\title{
Las cuevas de la Sierra de Atapuerca y el uso humano del paisaje kárstico durante el Pleistoceno (Burgos, España)
}

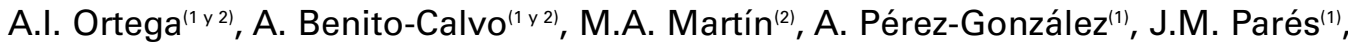 \\ J.M. Bermúdez de Castro(1), J.L. Arsuaga ${ }^{(3 \times 4)}$ y E. Carbonell ${ }^{(5,6 y 7)}$
}

(1) Centro Nacional de Investigación sobre la Evolución Humana (CENIEH), Paseo de la Sierra de Atapuerca 3, E-09002 Burgos, España

(2) Grupo Espeleológico Edelweiss (GEE). Diputación Provincial, E-09071 Burgos, España.

(3) Centro Mixto Universidad Complutense de Madrid - Instituto de Salud Carlos III de Evolución y Comportamiento Humanos (UCM-ISCIII), E-28029 Madrid, España.

(4) Departamento de Paleontología, Facultad Ciencias Geológicas, Universidad Complutense de Madrid (UCM), E-28040 Madrid, España.

(5) Institut Catala de Paleoecologia Humana i Evolucio Social (IPHES), E-43007Tarragona, España.

(6) Departament d'Història i Història de I'Art, Universitat Rovira i Virgili (URV), Campus Catalunya, E-43002 Tarragona, España.

(7) Institute of Vertebrate Palaeontology and Palaeoanthropology (IVPP) 100044 Beijing, China

\section{RESUMEN}

El karst de la Sierra de Atapuerca representa un interesante sistema multinivel, inactivo y heredado de antiguos niveles de base plio-pleistocenos, que alberga los enclaves prehistóricos más importantes para el conocimiento del poblamiento antiguo en Eurasia, y que fue declarado Patrimonio de la Humanidad en 2000 por Ia UNESCO. Estas cuevas se originan a partir de conductos subhorizontales con paleodrenajes en sentido S$\mathrm{N}$, localizándose la zona de descarga en la cabecera del río Pico. Los conductos están organizados en tres niveles principales que aparecen colgados entre 90 y 60 m sobre el actual cauce del río Arlanzón, coincidiendo con los niveles de base generados por sus terrazas fluviales T2, T3 y T5. La incisión fluvial liberó de las aguas los conductos superiores mientras se excavaban los niveles inferiores del karst. Las cuevas que iban quedando accesibles fueron utilizadas por la fauna y los homininos, conservando un registro arqueo-paleontológico de más de 1,2 Ma.

Palabras clave: espeleogénesis, karst, ocupación humana, Pleistoceno, Sierra de Atapuerca.

\section{The caves of Sierra de Atapuerca and human use of the karst landscape during the Pleistocene (Burgos, Spain)}

\begin{abstract}
The Sierra de Atapuerca karst is an interesting inactive multilevel cave system, which is the result of the action of ancient base levels during the Plio-Pleistocene. It contains one of the most important prehistoric sites for the understanding of old settlements in Eurasia, and was listed by the UNESCO World Heritage Site in 2000. These caves were formed on the bases of sub-horizontal conduits with SN paleo-drainage, with the discharge zone located in the head of Pico river. The conduits are organized into three main levels that appear perched between 90 and $60 \mathrm{~m}$ above the current course of the Arlanzón River, coinciding with the base levels generated mainly by the fluvial terraces T2, T3 and T5. Incision of the water table released the upper ducts from the action of water while the lower levels were being developed in the phreatic zone. The vadose caves would have been used by wildlife and hominids for more than $1.2 \mathrm{Ma}$, preserving the important archaeo-paleontological records of the Early and Middle Pleistocene.
\end{abstract}

Key words: human occupation, karst, Pleistocene, Sierra de Atapuerca, speleogenesis. 
Ortega, A.I., et al., 2018. Las cuevas de la Sierra de Atapuerca y el uso humano del... Boletín Geológico y Minero, 129 (1/2): $83-105$

\section{ABRIDGED ENGLISH VERSION}

\section{Introduction}

The Sierra de Atapuerca is $15 \mathrm{~km}$ east of the city of Burgos (northern Spain). It is located in the north-eastern area of the Cenozoic Duero Basin and south of the Bureba Corridor, which connects with the Ebro Basin and the Cantabrian and Iberian Ranges. This range forms the last foothills of the Iberian Chain, and corresponds to an overturned anticline composed of Mesozoic rocks.

\section{Atapuerca Karst System}

The karst in this hill was formed in a 40-70 m thick sequence of the Cretaceous limestone and dolomites, and shows the main geomorphological characteristics traits of a medium mountain karst. It exhibits an endokarst developed in the southwest flank of the San Vicente hill (1085 $\mathrm{m}$ a.s.l.). The accessible caves include $4.7 \mathrm{~km}$ of passages, among which are the Cueva Mayor, the Cueva del Silo system and the Peluda and Compresor caves. About fifty more fossil caves were discovered in the $20^{\text {th }}$ century when a railway line cut through the limestone, amongst which are the Sima del Elefante, Gran Dolina and Galería cave entrances (Ortega et al., 2012).

The caves consist of multilevel systems of three inactive sub-horizontal passages perched about 90,70 and $60 \mathrm{~m}$ above the current course of the Arlanzón river (Ortega et al., 2013, Benito-Calvo et al., in this volume). The geomorphological evolution analyses of the Sierra de Atapuerca landscape (Benito-Calvo, 2004) and the study of the karst systems (Ortega, 2009) reveal a connection between the karst formation and the evolution of the nearby Arlanzón river. This was an old discharge karst area in relation with paleo-springs at the headwater of the Pico River (Figs. 1-4).

\section{The Upper Level}

This level is the largest (Figs. 1B,5), with a length of 1,000 $\mathrm{m}$ and an average width of $10 \mathrm{~m}$ and a height of 15 $\mathrm{m}$. The passage in the Cueva Mayor exhibits sub-circular shaped roofs at 1,015-1,020 m a.s.l., while some dissolution chimneys can reach altitudes of 1,025-1,030 m. This level is a paragenetic conduit with phreatic section situated about $+90 \mathrm{~m}$ above the modern base level, coinciding with the Lower Páramo Plateau (1,0201,025 $\mathrm{m}$ a.s.l), and the Arlanzón fluvial terrace T2 (+82-91 $\mathrm{m})$. This indicates the relative stability of the water table over a long time span, favouring the formation and enlargement of the passages.

The drop of the water-table enabled the development of flowstone on the surface of the conduit. The evolution of the drop, marked by the T3 Arlanzón river terrace $(+70-78 \mathrm{~m})$, allowed the formation of passages with keyhole cross-sections in their distal sectors (Ortega et al., 2013). No trace of human presence in this level has ever been recorded to belong to this period of time.

\section{The Intermediate Level}

The second level is located in the Cueva Mayor at 1,000-1,005 $\mathrm{m}$ a.s.l. It is smaller than the upper level (700 $m$ long, 6-10 $m$ wide and 2-4 $m$ high), and has shifted to the west (Figs. 1B, 5). This passage begins in the south and is associated with a rising flow following fractures in the contact between the Cretaceous carbonates and Miocene marls. The passage continues to the confluence of the Propiedad and Valhondo valleys, where the resurgences of Pico river is located: here are found the Elefante cave (Cueva Mayor) and Gran Dolina-Penal (Trinchera sector) (Fig. 4).

These caves are phreatic passages related to the stability period of the Arlanzón terrace T3 (+70-78 m), which was developed about 1.14 \pm 0.13 Ma years ago (Moreno et al., 2012). Another drop in the water table deepened the bottom of the conduits, allowing the input of sediments. The first occupation of humans and animals is recorded in the Sima del Elefante 1.2 Ma ago (Carbonell et al., 2008). This occupation is one of the oldest settlements in Europe.

The period of the base level stability of the T4 terraces $(+60-67 \mathrm{~m})$ led to the formation of the Galería cave whose phreatic roof is $995 \mathrm{~m}$ a.s.l., and the vadose morphologies of the passages of the second level, such as the Gran Dolina, Elefante and Sala de los Cíclopes in the Cueva Mayor. This terrace is the last to be formed in the Early Pleistocene, and it is related to the stratigraphic sequences of TD7 (Dolina) and TE16-17 (Elefante), where the altitude is similar. Little evidence of human and animal activity has been found in these caves. However, traces of flooding are evident in the karst.

The Lower Level 
Ortega, A.l., et al., 2018. Las cuevas de la Sierra de Atapuerca y el uso humano del... Boletín Geológico y Minero, 129 (1/2): 83-105

This is the smallest level and displays a new shift westwards (Figs. 1B, 5). At the extremes of this level there are two mazes: the Silo (south) and Compresor (north) caves. The formation of the Peluda cave was linked to the main conduit of the Silo cave. The Sima de los Huesos, in the Cueva Mayor, is a $15 \mathrm{~m}$ deep shaft with ascending scallops. This level developed at 985-990 $\mathrm{m}$ a.s.l., and is perched at $+58-51 \mathrm{~m}$ above the Arlanzón river, related to the T5 terraces $(+50-58 \mathrm{~m})$. A fluvial input from the Arlanzón River has been documented inside Silo-Peluda system. This triggered the development of this level, and caused the subsidence and deformation of the Elefante sedimentary units (TE7 to TE17) (Ortega, 2009). The T5 terrace is the first to show a normal polarity, attached the Middle Pleistocene period, and can be related to the sediments above the fluvial deposits in the Silo cave, which also show normal polarity (Parés et al., in revision).

\section{Inactive Karst}

The T7 terrace $(+38-42 \mathrm{~m})$ represents the last interaction with the multilevel system, related to the enlargement of the little passages in the Silo, Peluda and Compresor caves $(978 \mathrm{~m}$ a.s.l.), and the incision of the fluvial deposits of the Silo-Peluda cave system. With the base level recorded by the T8 terrace (+26-35 m), the multilevel cave system became totally disconnected from and perched above the Arlanzón river network.

A third cultural phase corresponds with the arrival of the hominids of the Middle Pleistocene around 500 ka ago, who used this territory intensively. Evidence of this can be found in the Sima de los Huesos, situated in the dark zone of the Cueva Mayor. The earliest Acheulean technology is recognised in the GII-III levels of Galería (GII-III), thanks to the collapses of the roof of the cave, between about 500 and 250 ka years ago. The base camp is identified in the TD10 of Dolina, with the local transition from Mode 2 to 3, around 300 ka ago. The last traces of human activity can be found in the Elefante (TE19). All this evidence reveals the significance of the human occupations. Stratigraphic and biostratigraphic studies have shown that entrance sectors in the intermediate level were filled to the roof in the late Middle Pleistocene (Ollé et al., 2013; Cuenca-Bescós et al., 2010; Rodríguez et al., 2011) and the Sima de los Huesos was isolated from the outside environment (Arsuaga et al., 2014). In the Upper Pleistocene, the karst of San Vicente hill became inactive and was completely perched, with only minimal animal and human activity being documented. A fourth phase of the general human occupation of the karst took place in recent prehistoric times, when all the open caves in San Vicente hill were used for diverse purposes (Ortega, 2009).

\section{Conclusions}

The Atapuerca cave system comprises various sub-horizontal passages of water table caves, with drainage directions from south to north, developing parallel to the anticline axis. Towards the north, the passages intersect the surface terrain, which must have caused resurgences or springs to form at the head of the River Pico during the Pleistocene. The passages are stepped on three main levels, perched around $+90 \mathrm{~m},+70 \mathrm{~m}$, and +60 $m$ above the modern Arlanzón river, coinciding with the water table levels generated by the fluvial terraces. These levels show a progressive displacement toward the west and record the episodic down-stepping of the regional base level. During this evolution, the caves have become progressively vadose, allowing the entrance of fauna and hominids, whose activities were preserved in Early and Middle Pleistocene sedimentary sequences filling the cave entrances, such as the Trinchera sites (Dolina, Galería and Elefante), or in the interior of the caves, such as the Cueva Mayor sites (Sima de los Huesos). Three cultural phases have been documented, in relation to the different arrivals of hominids in western Europe. These migrations have made use of this karst territory in different ways: in the first occupation, during the pre-Jaramillo period the presence is very sporadic; however, it intensified during the end of Early Pleistocene, according to the Homo antecessor activity remains found in the Gran Dolina. The lack of archaeological record, along with evidence of cave clogging (Dolina) suggests the absence of humans. The third phase corresponds to the hominin recolonisation during the Middle Pleistocene, with an intense use of the whole caves and the surrounding territory.

\section{Introducción}

El escenario sobre la ocupación humana en Europa se ha visto alterado en los últimos 20 años por el descubrimiento en el sur de Europa de nuevos hallazgos, que han reavivado el debate sobre la antigüedad de los primeros asentamientos y los patrones de disper- sión y ocupación de Eurasia durante el Pleistoceno inferior y medio (Carbonell et al., 2010; Leroy et al., 2011; Muttoni et al., 2011; Bermúdez de Castro y Martinón, 2013). Este escenario muestra las primeras dispersiones humanas y la temprana colonización de Europa al área circunmediterránea (p.ej. MacDonald et al., 2013; Palombo, 2013; Rodríguez et al., 2011) y 
enlaza el concepto de adaptación de los homininos a las condiciones climáticas de las latitudes medias de Europa, barrera biogeográfica que sólo será superada con las nuevas migraciones de finales del Plesitoceno inferior y medio (Dennell et al., 2011; Mosquera et al., 2013, Bermúdez de Castro and Martinón-Torres, 2013).

El paisaje mediterráneo es un relieve montañoso complejo, fragmentado y muy accidentado, caracterizado por un mosaico de medios kársticos, con ejemplos de amplias llanuras o grandes valles que actúen como vías naturales de comunicación. Sus cuevas, abrigos y dolinas fueron usadas por los carnívoros y los homininos en sus rutas migratorias hacia el oeste de Europa, y actuaron como trampas naturales para los sedimentos, la fauna y los humanos, por lo que en su interior conservan una gran variedad de registros arqueo-paleontológicos (Brugal et al., 2005). En con- secuencia, la investigación de los sistemas kársticos permite desarrollar patrones de ocupación en la Prehistoria y caracterizar el paisaje social en relación a las limitaciones medioambientales del Pleistoceno.

\section{Contexto geográfico y geológico}

La Sierra de Atapuerca se sitúa a unos $15 \mathrm{~km}$ al Este de la ciudad de Burgos, en el sector nororiental de la Cuenca del Duero y en el límite suroeste del Corredor de la Bureba, paso natural entre la Cordillera Cantábrica y el Sistema Ibérico que comunica las cuencas cenozoicas del Ebro y del Duero (Fig. 1). Constituye un relieve estructural, perteneciente a la orla mesozoica del Sistema lbérico, de unos $14 \mathrm{~km}^{2}$ de extensión y subdividido en los montes de

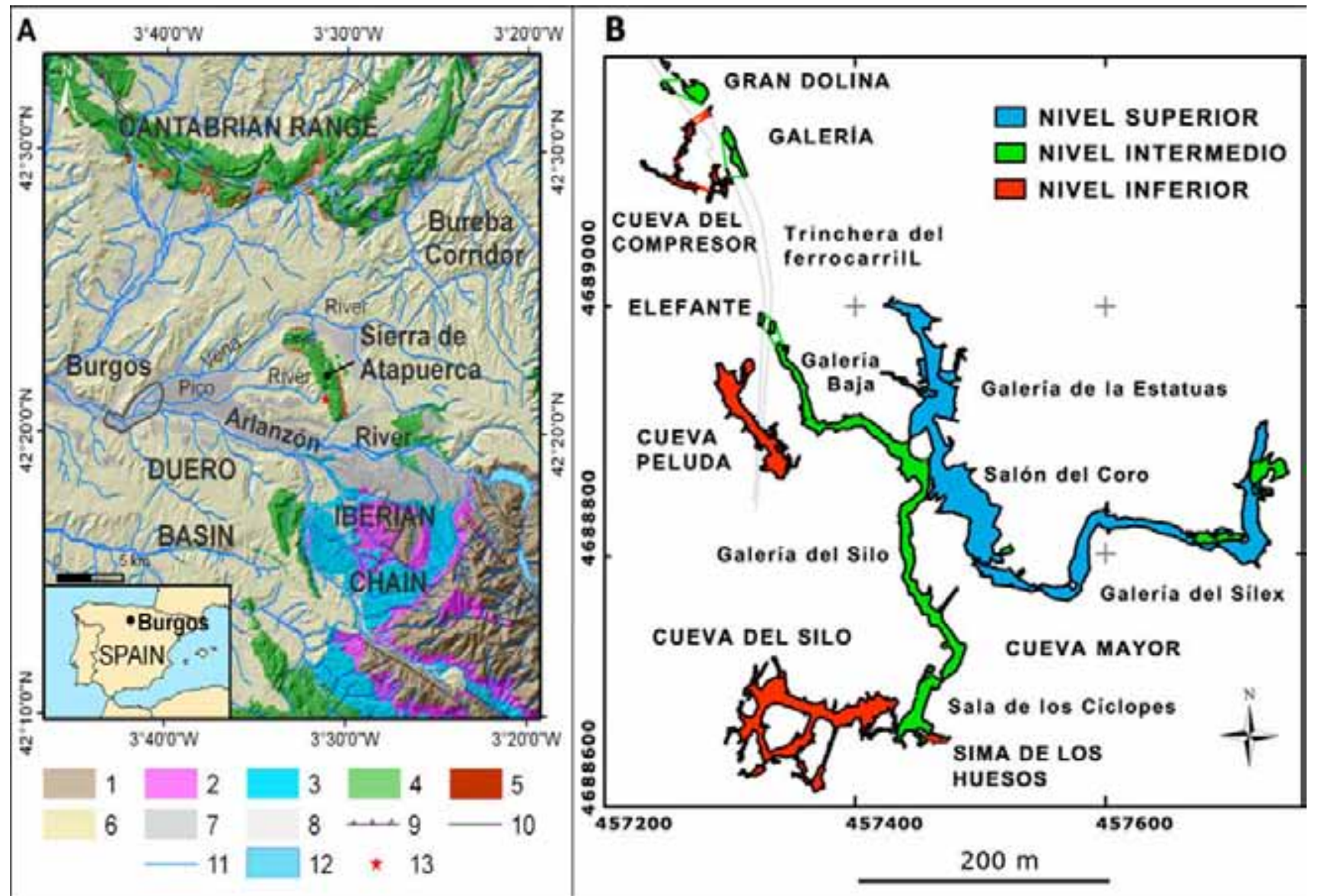

Figura 1. A) Localización geográfica y marco geológico. Leyenda; 1, Paleozoico; 2, Triásico; 3, Jurásico; 4, Cretácico; 5, Paleógeno; 6 , Neógeno; 7, Pleistoceno; 8, Holoceno; 9, Cabalgamiento; 10, Falla; 11, Red de drenaje; 13, Sierra de Atapuerca (según Benito-Calvo et al., 2008). 1B) Mapa del sistema de cavidades de la Sierra de Atapuerca (según Ortega, 2009).

Figure 1. A) Geographical location and geological framework. Legend: 1, Paleozoic; 2, Triassic; 3, Jurassic; 4, Cretaceous; 5, Paleogene; 6, Neogene; 7, Pleistocene; 8, Holocene; 9, Thrust; 10, Fault; 11, Drainage network; 13, Sierra de Atapuerca (after Benito-Calvo et al., 2008). 1B) Map of the Atapuerca cave system (after Ortega, 2009). 
Matagrande (1082 m s.n.m.) y San Vicente (1085 m s.n.m.). Representa un anticlinal tumbado con vergencia NE y dirección NNO-SSE, compuesto principalmente por calizas y dolomías del Cretácico superior (Pineda y Arce, 1997), que aparece aislado por sedimentos neógenos. Su extremo NO aparece fallado y desventrado, aflorando los materiales del núcleo (Jurásico y Cretácico inferior).

Forma un paisaje escalonado definido por la formación de distintas morfologías que han controlado la evolución del nivel de base local, caracterizado por cuatro superficies de erosión (SE1-SE4) desarrolladas durante el Neógeno, disectadas por los valles fluviales durante el Cuaternario, dando lugar a la formación de una secuencia de terrazas fluviales (Benito-Calvo y Pérez-González, 2015).

La incisión fluvial está jerarquizada por el río Arlanzón, que fluye al sur de la sierra en sentido E-O y del que son tributarios los ríos Vena y Pico. El Vena la drena por el norte mientras que el Pico, que nace en la propia sierra, fue alimentado por las paleosurgencias kársticas de su ladera SO. El Arlanzón, que discurre actualmente en la perpendicular del sistema kárstico a $936 \mathrm{~m}$ s.n.m., presenta un sistema de 14 terrazas fluviales y la llanura de inundación, correspondiendo las terrazas T1 a T4 al Pleistoceno inferior, la T5 a T10 al Pleistoceno medio, la T11 a T13 al Pleistoceno superior y la T14 al Holoceno (BenitoCalvo et al., en este volumen).

La evolución regional de los niveles de base ha controlado la karstificación en la Sierra de Atapuerca, centrada durante el Pleistoceno en el desarrollo del sistema multinivel de Torcas, en el sector occidental del San Vicente (Ortega et al., 2013). El descenso de los niveles de base durante el Pleistoceno inferior liberó de las aguas las cavidades del karst, permitiendo el acceso a los humanos y animales que han dado lugar a los excepcionales yacimientos de la Sierra de Atapuerca.

\section{Metodología}

El estudio del karst de Atapuerca se ha basado en el análisis de diversas bases de datos espaciales a partir de la detallada exploración y estudio de las morfologías kársticas. En el trabajo de campo se utilizaron equipos de topografía espeleológica (brújula y clinómetro Suunto, cinta métrica y distanciómetro láser) (Martín, 2000). Se establecieron poligonales y bases de referencia en el karst, un itinerario de vértices en Trinchera, así como una red local geodésica de vértices, mediante GPS, creando una Red Regente de Alta Precisión y un sistema de coordenadas único (Pérez, 2011).
Han sido utilizadas plataformas SIG (ArcGIS 10.2) y CAD para la georeferenciación de todos los datos y la necesaria integración de la información geológica, geomorfológica y espeleológica (Benito Calvo, 2004; Ortega, 2009; Benito-Calvo y Pérez-González, 2015). La identificación de los sectores paleosurgentes se utilizó para establecer la posición de los paleoniveles de base que controlaron el desarrollo de los conductos (Palmer, 2007). La caracterización de diferentes niveles subhorizontales permitió inferir la secuencia espeleogenética. Finalmente se propuso un modelo simplificado de la evolución del karst, basado en la evolución de las morfologías superficiales y de los excepcionales registros cronoestratigráficos de los yacimientos de la Sierra de Atapuerca.

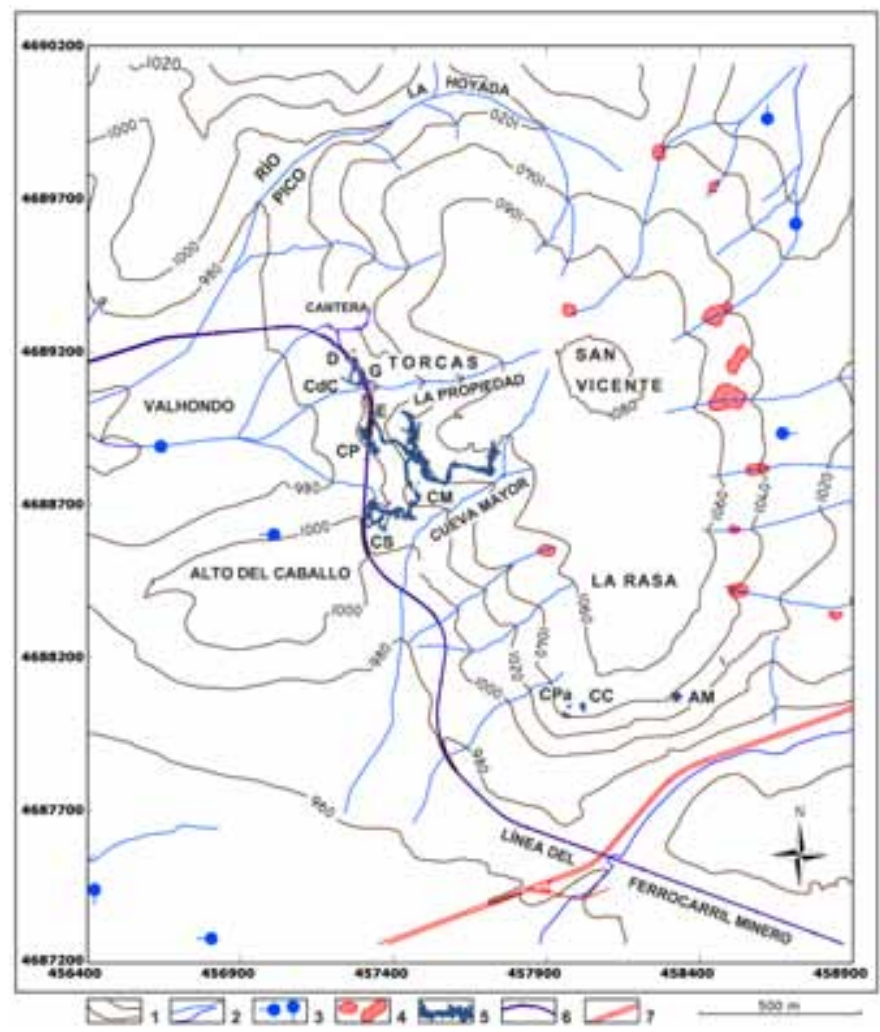

Figura 2. Situación de las cuevas en el monte de San Vicente. Leyenda: (1) Curvas de nivel; (2) Superficie de drenaje; (3) Manantiales; (4) Dolinas; (5) Cuevas; (6) Trazado del Ferrocarril; (7) Carretera; AM: Abrigo del Mirador; CC: Cueva Ciega; CdC: Cueva del Compresor; CM: Cueva Mayor; CS: Cueva del Silo; CP: Cueva Peluda; CPa: Cueva La Paredeja; D: Gran Dolina; E: Sima del Elefante; G: Galería (Modificado de Ortega et al., 2013).

Figure 2. Location of the caves in the San Vicente hill. Legend: (1) contour lines; (2) surface drainages; (3) springs; (4) dolines; (5) caves; (6) railway line; (7) road; AM: Mirador Rock-Shelter; CC: Cueva Ciega; CdC: Cueva del Compresor; CM: Cueva Mayor; CS: Cueva del Silo; CP: Cueva Peluda; CPa: Cueva La Paredeja; D: Dolina site; E: Elefante site; G: Galería site (modified from Ortega et al., 2013). 


\section{El karst de la Sierra de Atapuerca}

La Sierra de Atapuerca muestra un karst de media montaña desarrollado en las calizas y dolomías del Cretácico superior y caracterizado por un importante desarrollo de morfologías subterráneas en contraposición con la escasa entidad de las formas exokársticas: lapiaces y dolinas. Las cavidades accesibles se localizan en el monte San Vicente, agrupadas en dos sectores diferenciados, el conjunto de La Rasa y el de Torcas (Fig. 2). Destaca este último por concentrar dos grandes sistemas paleosurgentes, con $4,7 \mathrm{~km}$ de conductos subhorizontales topografiados y más de medio centenar de cuevas colmatadas, que muestran un karst inactivo y colgado sobre los cursos actuales de los ríos Arlanzón y Pico (Fig. 3) (Ortega, 2009; Ortega et al., 2013).

Son cavidades formadas en régimen freático, de tipo paragenético, con fuerte control estructural a favor del flanco oeste del anticlinal, donde los estratos presentan direcciones en torno a $\mathrm{N} 170^{\circ} \mathrm{E}$ y buzamientos de $17-20^{\circ}$, documentando una coincidencia bastante aproximada entre el sistema de fracturas del sector de Torcas y las direcciones preferentes de las galerías del sistema endokárstico (Figs. 4 y 5) (Eraso et al., 1998; Ortega, 2009).

\section{Las cavidades de La Rasa}

En el borde meridional del San Vicente, término de La Rasa (Fig. 2 y 5.C), destacan las cuevas Ciega y Paredeja y el relleno fosilífero CR6 por formar parte de un mismo conducto subhorizontal de $110 \mathrm{~m}$, situado a unos 1050-1055 m de altitud. Se localiza por debajo de la superficie de erosión del Mioceno medio (SE2, 1074-1050 m s.n.m., Benito-Calvo y PérezGonzález, 2015) y a $115 \mathrm{~m}$ sobre el curso actual del río Arlanzón (Ortega et al., 2013), representan al nivel más elevado del karst. El Abrigo del Mirador y el conducto colmatado por margas (CR7), situados a unos 1030 m s.n.m. y a unos $95 \mathrm{~m}$ sobre el curso del río Arlanzón, se relaciona con la formación de la SE4 (1036-1031 m s.n.m.), de edad plio-pleistocena (Ortega, 2009; Benito-Calvo et al., 2008).

\section{El karst del término de Torcas}

La ladera occidental del San Vicente constituye el núcleo de karstificación más importante de la Sierra de Atapuerca y uno de los más significativos de la Depresión del Duero (Martín et al., 1981). Está compuesto por el sistema de Cueva Mayor-Cueva del Silo
$(3,7 \mathrm{~km})$, cueva Peluda (380 m) y cueva del Compresor (475 m), así como medio centenar de cavidades colmatadas en la Trinchera del Ferrocarril y frentes de canteras, entre las que destacan los yacimientos de Sima de Elefante, Gran Dolina o Galería (Figs. 4 y 5). El karst está delimitado por el valle de Cueva Mayor al sur, el valle de la Hoyada al norte, la cumbre del San Vicente al este y el Valle de Valhondo al oeste (Fig. 2). El valle de La Propiedad aísla el sistema de Cueva Mayor-Silo de los conductos de Trinchera.

Sus cavidades tienen una génesis hipogénica, estando originadas por aguas ascendentes que fluyen, desde un acuífero semiconfinado hasta alcanzar el nivel freático local, a favor de planos de debilidad. Más de 30 de estas chimeneas ascendentes se localizan en el borde meridional de las calizas, en el contacto con las margas neógenas que recubren el escalón del valle de Cueva Mayor. Una vez alcanzado el equilibrio hidrostático, las aguas fluían hacia el NNO por grandes conductos subhorizontales controlados por antiguas fases de estabilidad del nivel de base (Ford y Williams, 2007). Los conductos se distribuyen en tres niveles principales, en tan sólo $52 \mathrm{~m}$ de desnivel total, con paleosurgencias escalonadas que emergen en su confluencia con los valles de La Propiedad y Valhondo (Ortega, 2009), drenando el acuífero kárstico en la cabecera del río Pico (Fig. 5).

Los conductos presentan un trazado inicial sinuoso hasta adaptarse a la dirección NNO, paralela a la estratificación y al eje del anticlinal. Muestran desplazamientos progresivos hacia el oeste, debido al encajamiento del río del Arlanzón. Este descenso propició la incisión vadosa de los pasajes, creando secciones tipo "ojo de cerradura" así como la formación de los niveles inferiores (Fig. 6) (Ortega et al., 2013). Por otra parte, estos descensos del nivel de base posibilitaron el acceso y utilización de las cavidades inactivas como refugios y cubiles a lo largo del Pleistoceno inferior y medio.

\section{Nivel superior: conducto Sílex-Estatuas de Cueva Mayor}

El nivel superior corresponde al conducto de mayores dimensiones del karst, formado por la galería del Sílex, el Portalón, el Salón del Coro y la galería de las Estatuas de Cueva Mayor. Este conducto tiene unos $1000 \mathrm{~m}$ de desarrollo, secciones de unos $10 \mathrm{~m}$ anchura por unos $15 \mathrm{~m}$ de altura y techos abovedados próximos a los 1015-1022 m s.n.m., situados a +79-86 m sobre el cauce del río Arlanzón, alcanzando algunas chimeneas los 1025-1030 m de altitud. Se inicia en el centro de la sierra, con un primer tramo zigzagueante 


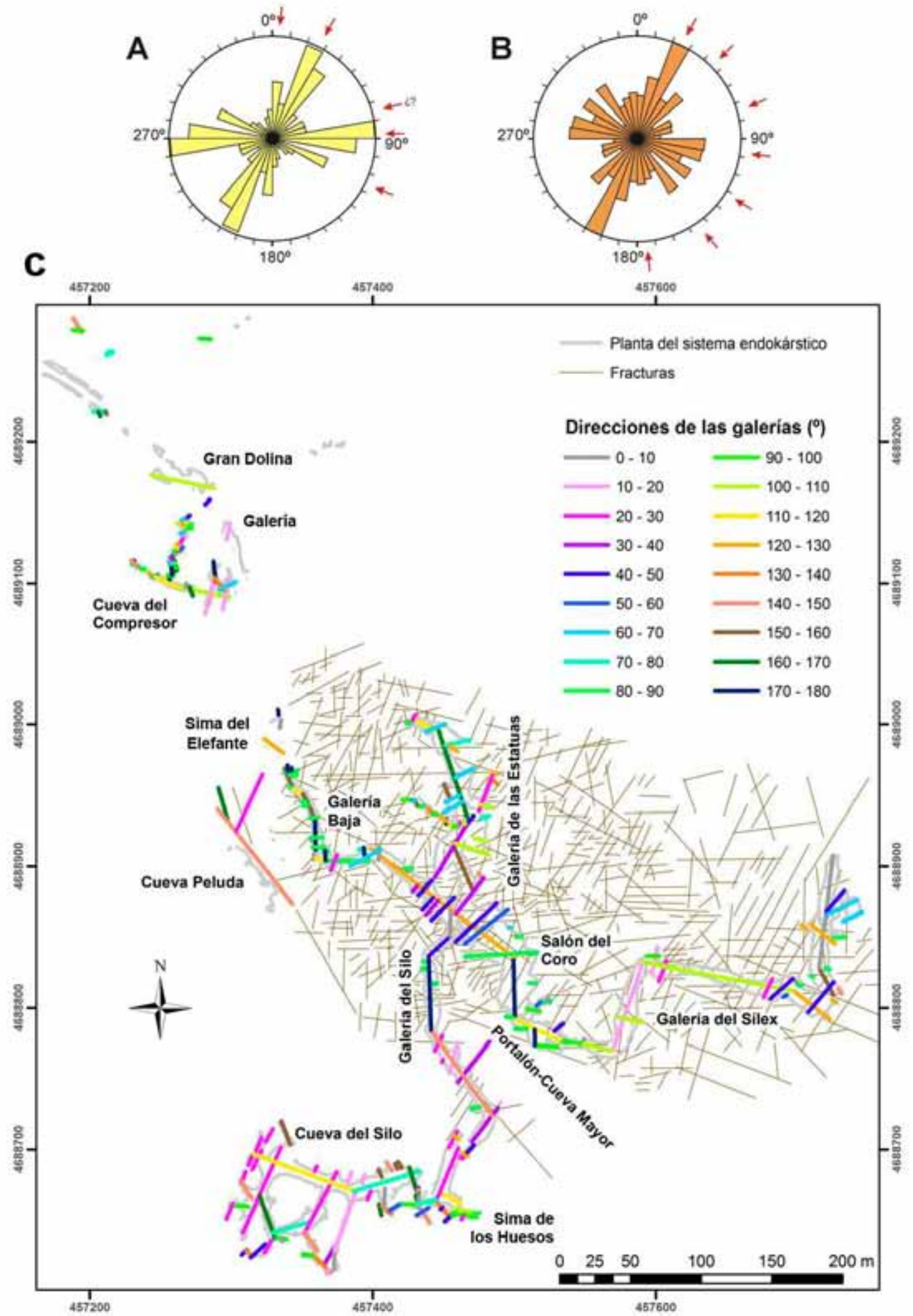

Figura 3. Diagramas de rosas de las direcciones de las fracturas (A) del flanco sudoeste de la Sierra de Atapuerca y (B y C) la dirección de las galerías del Sistema kárstico deTorcas-Cueva Mayor. Porcentajes calculados en función del desarrollo longitudinal (basado en Ortega, 2009).

Figure 3. Rose diagrams of the direction of fractures: A) direction of fractures in anticlinal flank (Torcas place). B and C) Direction of passages within the Torcas-Cueva Mayor karst system. Percentages estimated following longitudinal development (based on Ortega, 2009). 

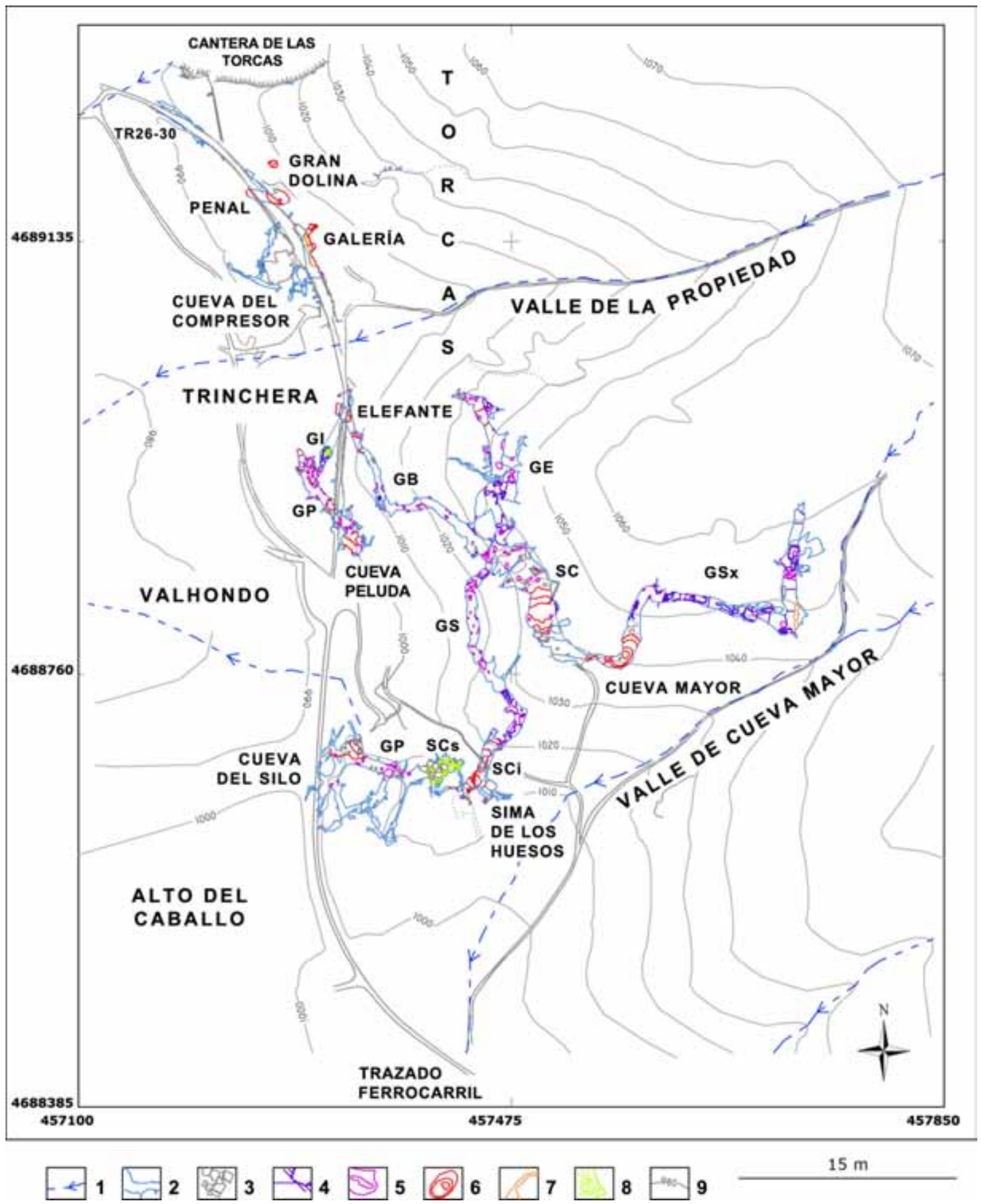

$15 \mathrm{~m}$

Figura 4. Mapa del sistema de cavidades de Atapuerca (ver situación en fig. 2). Leyenda: (1) Red de drenaje; (2) Cuevas; (3) Bloques; (4) Espeleotemas erosionados; (5) Espeleotema; (6) Facies alóctonas; (7) Facies autóctonas; (8) Gravas fluviales; (9) Margas; GSx: Galería del Sílex; SC: Salón del Coro; GE: Galería de las Estatuas; SCi: Sala de los Cíclopes, GS: Galería del Silo; GB: Galería Baja; SCs: Sala del Caos; GP: Galería Principal; GI: Galería inferior. (Topografía espeleológica del Grupo Espeleológico Edelweiss, según Ortega, 2009).

Figure 4. Map of the Atapuerca cave system (see location in Fig. 2). Legend: (1) drainage network; (2) caves; (3) blocks; (4) eroded speleothem; (5) speleothem; (6) allochthonous facies; (7) autochthonous facies; (8) fluvial gravels; (9) marls; GSx: Galería del Sílex; SC: Salón del Coro; GE: Galería de las Estatuas; SCi: Sala de los Cíclopes, GS: Galería del Silo; GB: Galería Baja; SCs: Sala del Caos; GP: Galería Principal; GI: Galería lower. (Speleological survey by Grupo Espeleológico Edelweiss, after Ortega, 2009). 


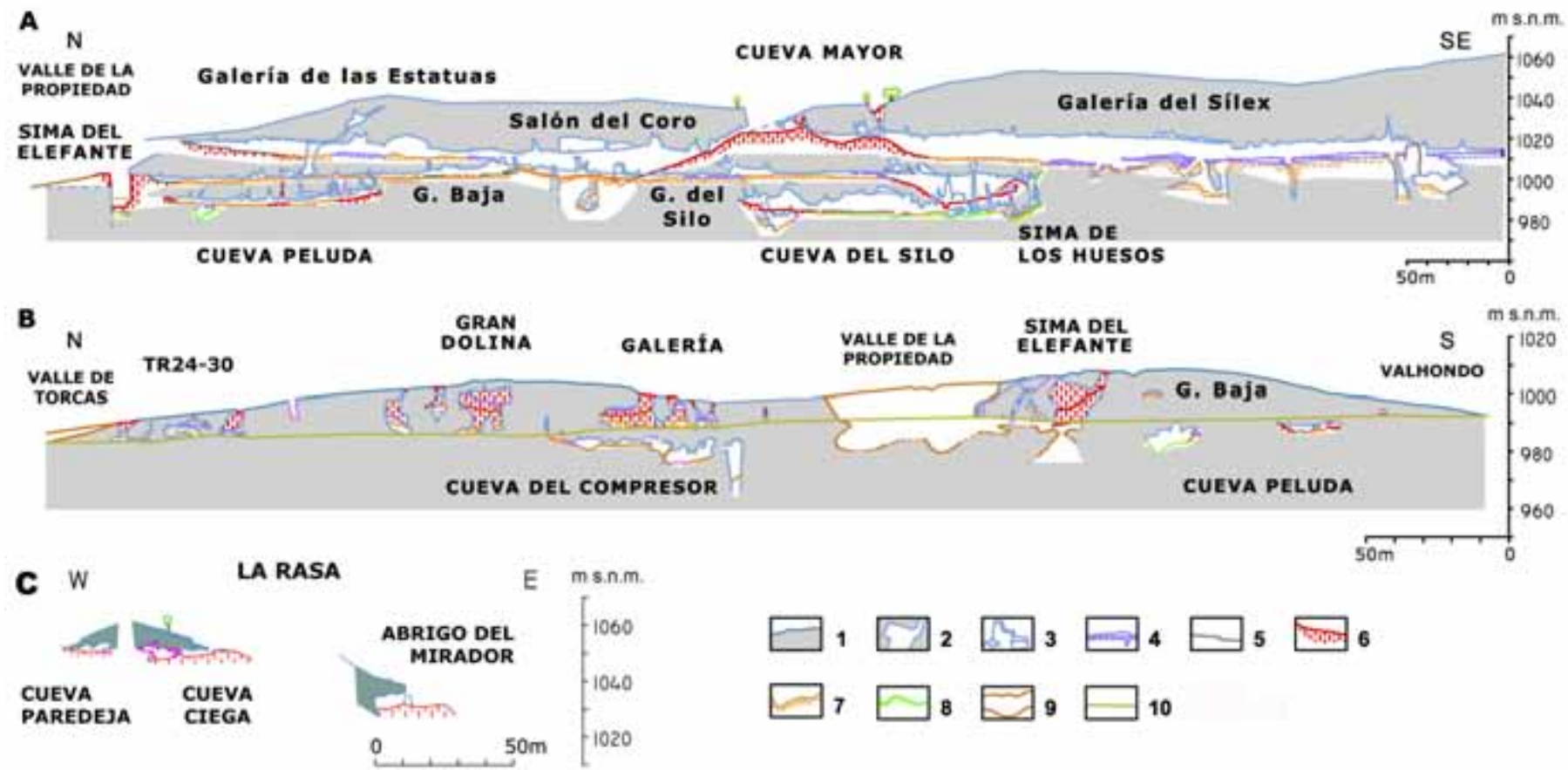

Figura 5. Perfiles longitudinales de las cavidades de la Sierra de Atapuerca. A) Sistema Cueva Mayor - Cueva del Silo y Cueva Peluda. B) Cuevas y conductos seccionados por la Trinchera del Ferrocarril. C) Cuevas de La Rasa. Leyenda: 1, Superficie del terreno; 2, Cuevas; 3, Blocques; 4, Espeleotemas erosionados; 5, Espeleotemas; 6, Facies alóctonas; 7, Facies autóctonas; 8, Gravas fluviales; 9, Valle; 10, Superficie de la Trinchera del Ferrocarril (Modificado de Ortega, 2009).

Figure 5. Longitudinal profiles of the Sierra de Atapuerca caves. A) Cueva Mayor - Cueva del Silo System and Cueva Peluda. B) Caves and passages exposed in the railway trench. C) La Rasa caves. Legend: 1, Land surface; 2, caves; 3, blocks; 4, eroded speleothem; 5, speleothem; 6, allochthonous facies; 7, autochthonous facies; 8, fluvial gravels; 9, valley; 10, base of railway trench (modified from Ortega, 2009).

hasta adaptarse a la ladera occidental, prosiguiendo con sentido NNO hacia el valle de La Propiedad, donde las aguas afloraban en la paleosurgencia más alta y antigua del karst. Muestra una morfología paragenética que evolucionó hacia tipos mixtos vadosos que, en algunos puntos, descienden hasta cotas del nivel intermedio (Figs. 4-6.A).

El Salón del Coro es el mayor volumen del sistema, formado por una sala de $100 \mathrm{~m}$ de largo por 30$40 \mathrm{~m}$ de ancho y entre 18 y $30 \mathrm{~m}$ de altura (Figs. 5.A y 7.B). Su morfología irregular está marcada por importantes colapsos de paredes y techos, a favor de planos de estratificación y fracturas. El punto más bajo de la sala se localiza en la base de un gran bloque a 985 m s.n.m., en cotas del nivel inferior del karst. La actual entrada de Cueva Mayor, el Portalón, se produjo por el colapso del techo a favor de una fractura E$\mathrm{O}$, proporcionando un acceso a la cavidad tanto para sedimentos como para los grupos de pastores y agricultores durante la Prehistoria reciente (Ortega et al., 2008).

El nivel superior aparece profusamente decorado. Presenta Destaca la continuidad de una potente cos- tra estalagmítica situada entre 1010 y 1014 m s.n.m., que presenta procesos de corrosión y reexcavación, y marca la posición de la superficie anterior al encajamiento vadoso (Fig. 5.A y 7.A). Datos magneto-estratigráficos procedentes del espeleotema erosionado del final de la galería de Estatuas, así como de los sedimentos finos que sustentan la costra en la Galería del Sílex, indican un evento de polaridad inversa relacionado con el cron Matuyama (Parés et al., in revision).

A este nivel corresponden diversas secciones de los frentes de canteras situadas a 1020 m s.n.m, como el yacimiento pleistoceno CR1, situado al NE de Gran Dolina (Fig. 4) (Ortega, 2009).

Nivel intermedio: conducto Cíclopes-Elefante de Cueva Mayor y Gran Dolina-Galería

El nivel intermedio es un conducto subhorizontal de génesis freática y evolución vadosa, localizado en Cueva Mayor entre 1000 y 1005 m s.n.m. y +64-69 m por encima del Arlanzón, con chimeneas ascendentes 

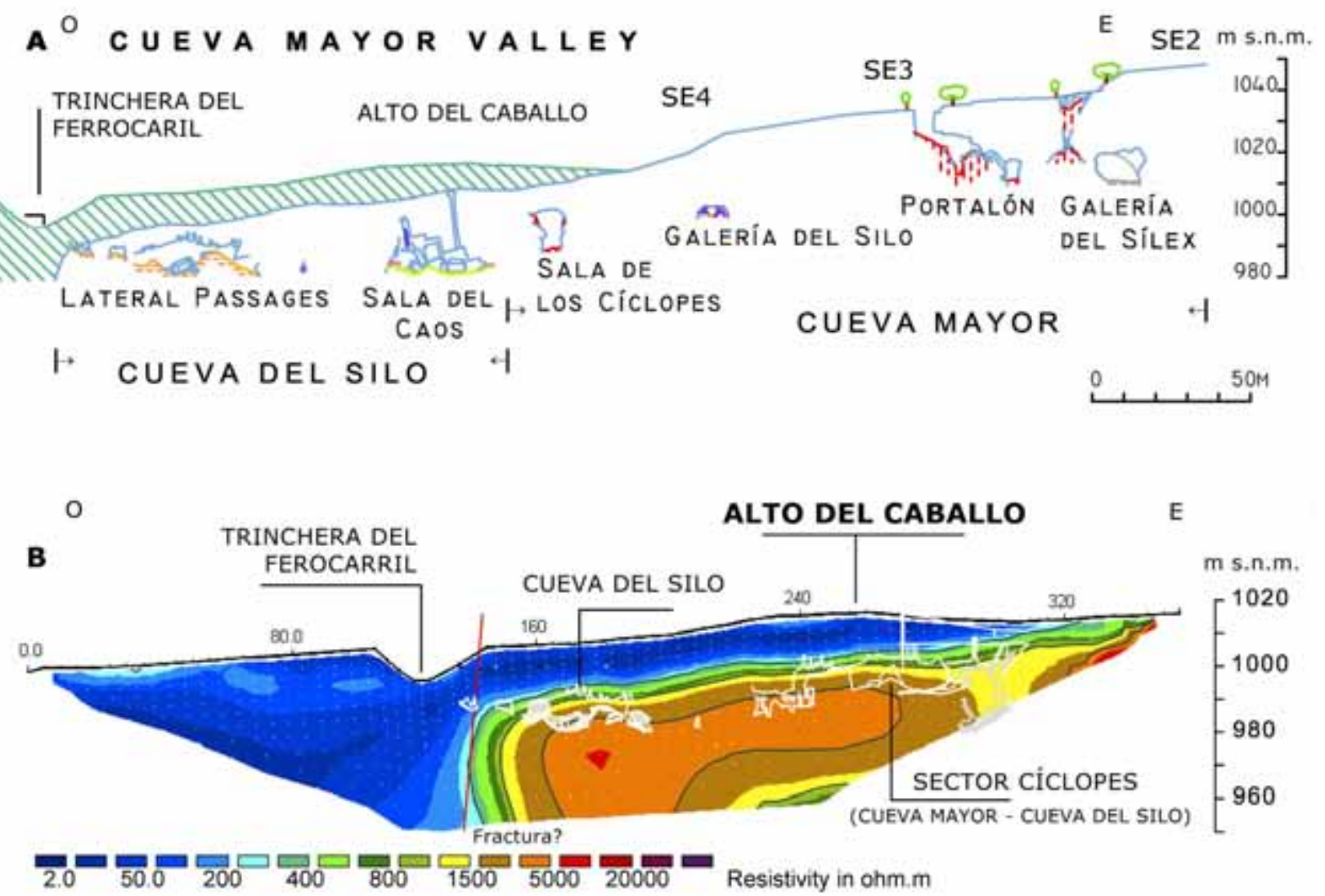

C $\mathrm{O}$

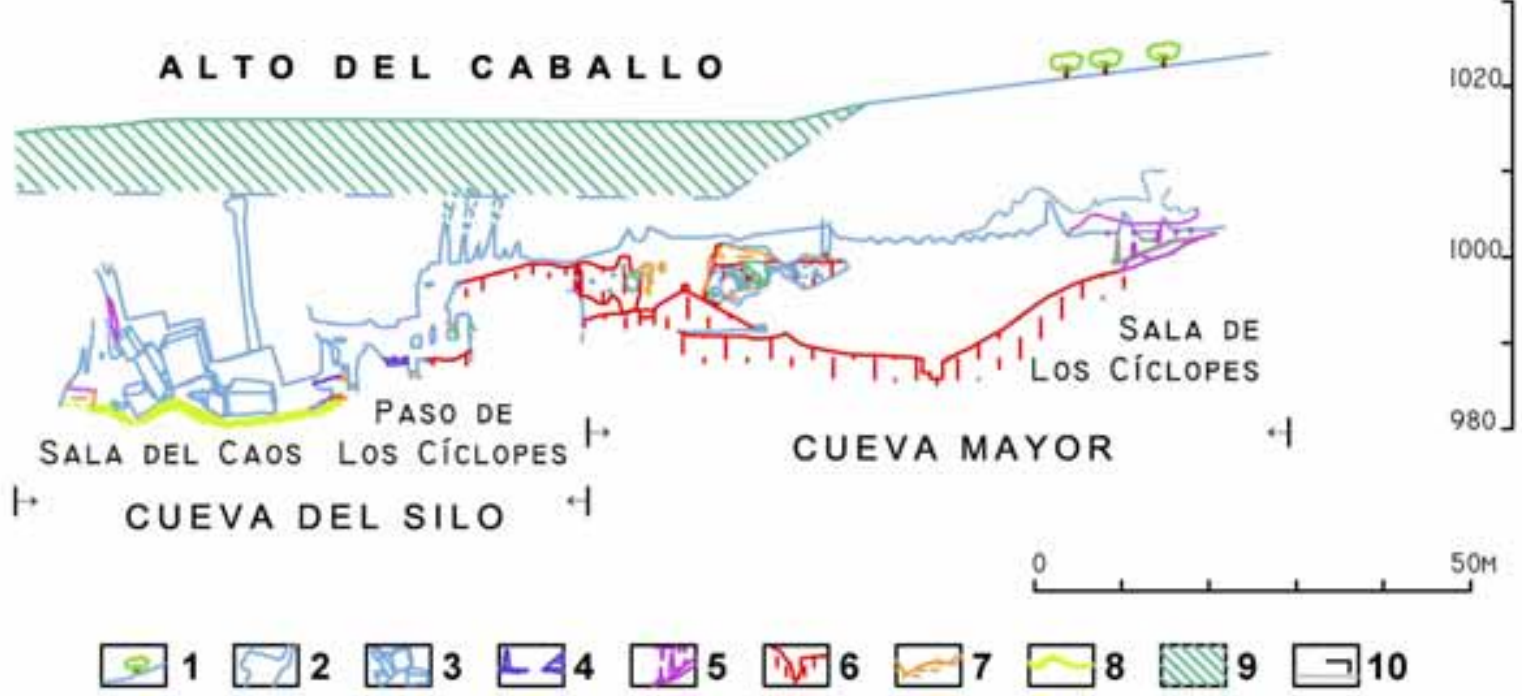

Figura 6. Secciones representativas (E-O) del Sistema de Cueva Mayor-Cueva del Silo y Cueva Peluda. A) Perfil paralelo al Valle de Cueva Mayor y sobre el Alto del Caballo. B) (A) Perfil de Tomografía de Resistividad Eléctrica. C) Sección del área de contacto entre los conductos de los niveles intermedio e inferior del sistema de Cueva Mayor-Cueva del Silo (Sector Cíclopes) (Basado en Ortega, 2009). (Ver leyenda en fig. 5, excepto 9 que son margas).

Figure 6. Representative sections (E-W) of the Cueva Mayor-Cueva del Silo and Cueva Peluda systems. A) Profile along the Cueva Mayor Valley and on top of Alto del Caballo. B) Electrical resistivity tomography profile. C) Detail of the connection area between the intermediate and lower passage levels in Cueva Mayor - Cueva del Silo System (Cíclopes Sector) (modified from Ortega, 2009). (See legend in Fig. 5, except 9 which are marls). 
que alcanzan los $1010 \mathrm{~m}$ s.n.m. Este nivel se origina en la sala de los Cíclopes y continúa por las galerías del Silo y Baja hasta la paleosurgencia de Elefante (Figs. 4-5 y 8.A). Presenta un trazado sinuoso de unos $700 \mathrm{~m}$ de desarrollo, paralelo al nivel superior aunque encajado unos $20 \mathrm{~m}$ y con secciones de $6-10 \mathrm{~m}$ de anchura y 2-4 $\mathrm{m}$ de altura. Al mismo nivel pertenecen los conductos desarrollados en las bases de las simas de las galerías del Sílex y Estatuas.

La sala de los Cíclopes se origina en los conductos ascendentes, a favor de planos de debilidad, de Sima de los Huesos y el paso de los Cíclopes, localizados en el límite de las calizas mesozoicas con las margas miocenas del valle de Cueva Mayor (Figs. 5-7.C) (Ortega et al., 2010, 2013). El conducto se dirige hacia el NNO hasta confluir en los valles de La Propiedad y Valhondo, donde las aguas resurgían por Sima del Elefante. Las secuencias sedimentarias de la fase de formación del conducto, observadas en los frentes de las secciones vadosas o antrópicas, muestran polaridades inversas (Parés et al., 2010, in revisión). El descenso del nivel de base generó la evolución del conducto en circulación libre, propiciando la entrada de sedimentación alóctona en el antiguo sector surgente de Sima del Elefante, en donde se documenta la ocupación humana más antigua de Europa relacionada con fases pre-Jaramillo (Carbonell et al., 2008).

La incisión vadosa desarrolló morfologías mixtas en los extremos del conducto, en la sala de los Cíclopes y Sima del Elefante, representando puntos de conexión con el nivel inferior (Figs. 5-7.C y 8.A). Este proceso produjo el encajamiento de los conductos y la erosión de los sedimentos que los colmataban, quedando sus testigos colgados en las paredes de la sala de los Cíclopes, entre los 1000 y $995 \mathrm{~m}$ de altitud, (Fig. 6.C). Entre estos sedimentos destacan las facies de arenas y limos fluviales, documentados también en las galerías del Silo y Baja, por presentar polaridades inversas atribuidas al cron Matuyama (Parés et al., 2010, in revision). La secuencia de Elefante muestra una importante fase de reactivación, con procesos de colapso y aportes de sedimentos procedentes del valle de la Propiedad que alcanzan la galería del Silo, en un momento de transición de Matuyama-Brunhes (Ortega, 2009; Parés et al., in revision).

Al nivel intermedio también pertenecen las cavidades situadas al norte del valle de la Propiedad, puestas al descubierto por la Trinchera del Ferrocarril minero y los frentes de las canteras (Ortega et al., 2012). Gran Dolina-Penal y Galería constituyen antiguas entradas fosilizadas, con importantes secuencias arqueo-estratigráficas (Figs. 4, 5.B y 8). La colmatación de estas cavidades no permite conocer el desarrollo tridimensional de sus conductos, pero la relación entre las secciones y el trabajo de excavación ayuda a extrapolar algunas direcciones. Los rellenos de Gran Dolina y Penal forman parte de un mismo conducto paleosurgente con sentido SE-NO, según marcan las huellas de corriente de sus paredes (Eraso et al., 1998; Ortega, 2009).

Gran Dolina cuenta con $18 \mathrm{~m}$ de altura vista en el frente de Trinchera (Figs. 4, 5.B y 8.B), más otros $8 \mathrm{~m}$ adicionales sondeados en 2014 en su base. Presenta una morfología mixta muy verticalizada, tipo "ojo de cerradura", con bóveda freática a unos 1000 m s.n.m. y cúpulas a unos $1003 \mathrm{~m}$ de altitud, mientras que el encajamiento vadoso aparece en torno a los $995 \mathrm{~m}$ s.n.m., cotas que estabilizan la bóveda de la cavidad de Galería, situada a unos $50 \mathrm{~m}$ al sur de Dolina. A partir del Pleistoceno inferior se produce la entrada de sedimentos de exterior y el uso humano del espacio.

Galería representa a una cavidad freática que aparece fosilizada y seccionada en la pared oriental de la Trinchera (Figs. 1.B, 4, 5.B y 8.C). Morfologías vadosas con encajamiento vertical parecen reconocerse en el sector norte. Está compuesta por la covacha de los Zarpazos, Galería y Tres Simas (Ortega, 2009). Una visión de conjunto parece revelar un volumen tridimensional que se amplía hacia el SO (parcialmente vaciado por las obras del ferrocarril). Diferentes colapsos del techo proporcionaron luminosidad y actuaron como trampas naturales a partir del Pleistoceno medio.

\section{Nivel inferior: cuevas del Silo, Peluda y Compresor}

El nivel inferior se caracteriza por conductos freáticos subhorizontales de pequeñas dimensiones, estabilizados en cotas de $982-990$ m s.n.m., entre +46 y $54 \mathrm{~m}$ sobre el actual nivel de base del río Arlanzón. En relación con los niveles superiores, continúa su progresivo encajamiento y desplazamiento lateral hacia el oeste, a favor de la ladera (Figs. 1.B y 4-6).

En las proximidades de los valles se desarrollan redes laberínticas, a favor de chimeneas ascendentes de origen hipogénico, cuyas aguas subterráneas drenaban al río Pico por paleosurgencias dispuestas en la cabecera del valle de Valhondo. Estas redes evolucionan en el límite de las calizas cretácicas, recubiertas por las margas miocenas, por lo que son frecuentes los colapsos de sus bóvedas y la entrada de los sedimentos neógenos (Ortega et al., 2012).

El nivel inferior está formado por Sima de los Huesos, y las cuevas del Silo, Peluda y Compresor, así como por el conjunto de conductos TR23-31, situados 

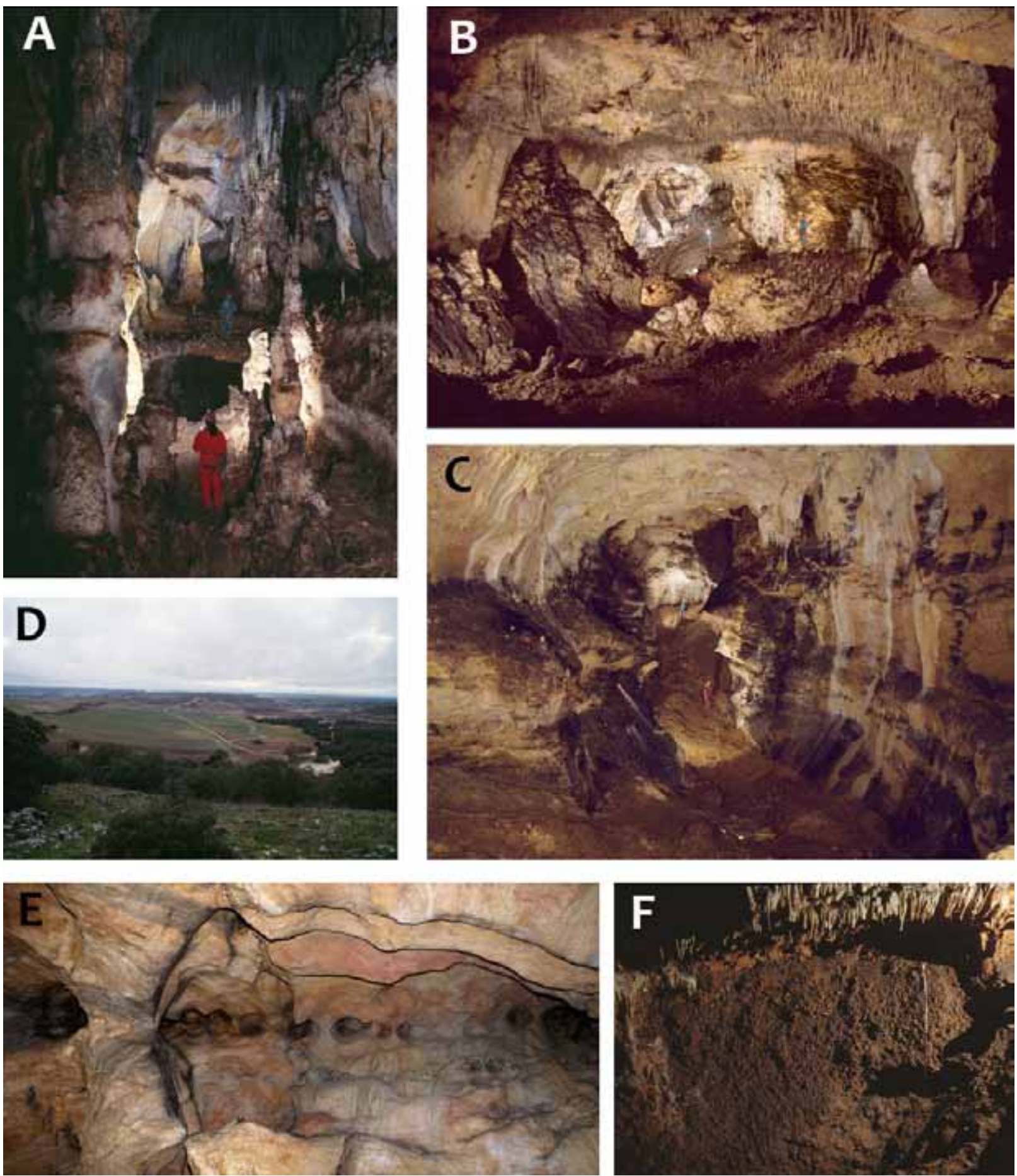

Figura 7. Ejemplos de la morfología del karst de Atapuerca. A) La incisión en la galería del Sílex (nivel superior) ha erosionado los sedimentos de interior y ha dejado colgado la costra estalagmítica. B) El salón del Coro es el mayor volumen del karst, con importantes bloques producto de colapso de las bóvedas y de las paredes. C) La sala de los Cíclopes (nivel intermedio) muestra una típica sección mixta "ojo de cerradura" con una importante erosión. D) Valle de Valhondo durante las inundaciones de marzo de 2001. E) Chimeneas ascendentes de cueva del Silo, con huellas de corriente que indican flujos ascendentes (nivel inferior). F) Depósitos fluviales de la galería inferior de cueva Peluda (Fotos: M.A. Martín, Grupo Espeleológico Edelweiss).

Figure 7. Examples of morphologies of the Atapuerca caves. A) The entrenchment of the gallery of the Silex (upper level), has eroded and has left the flowstone perched. B) The Salón del Coro is the largest chamber, with significant breakdown deposits derived from the roof and walls. C) The Sala de los Cíclopes (intermediate level) shows a typical keyhole section with significant erosion. D) temporal stream in Valhondo valley during flooding in March 2001. The rising chimneys of the Silo cave, with scallops indicating upward flow (lower level). F) Fluvial deposits of the lower gallery of the Peluda cave (Photos: M.A. Martín, Grupo Espeleológico Edelweiss). 
al final de Trinchera. Este nivel se inicia en el borde meridional del karst, junto al valle de Cueva Mayor, con los conductos ascendentes de Sima de los Huesos de Cueva Mayor, el paso de los Cíclopes y la sala del Caos de cueva del Silo, cuyas bóvedas alcanzan la sala de los Cíclopes ( nivel intermedio) (Fig. 6). Sima de los Huesos es una chimenea ascendente de $15 \mathrm{~m}$ de desnivel, con un pequeño conducto subhorizontal a 983-984 $\mathrm{m}$ de altitud. (Ortega, 2009).

Cueva del Silo presenta mayor complejidad y desarrollo (unos $1000 \mathrm{~m}$ ), existiendo más de 30 chimeneas ascendentes, de entre 10 y $20 \mathrm{~m}$, que alcanzan los 1003-1008 m de altitud y superan las bóvedas del segundo nivel (Figs. 4-7.E). Estas morfologías sugieren la importancia del ascenso de las aguas subterráneas en la génesis de la cavidad y la presencia de estos flujos en el entorno de El Silo de Valhondo (Eraso et al., 1998), indican que esta boca funcionó como paleosurgencia.

El conducto principal, de unos $130 \mathrm{~m}$ y sentido ESE-ONO, está formado por la sala del Caos y la galería Principal, a la que confluyen ortogonalmente conductos menores formando un enrejado con predominio de las fracturas $\mathrm{N} 110-130^{\circ} \mathrm{E}$ y N20-40 ${ }^{\circ} \mathrm{E}$ (Figs. 3 y 4) (Ortega, 2009). La presencia de colapsos del techo a favor de planos de debilidad incrementa el tamaño del conducto (Fig. 6.C). Incisión vadosa aparece en las zonas de conexión de las galerías laterales con el eje principal, con claros ejemplos de secciones mixtas. Prospecciones geofísicas muestran el desarrollo del conducto principal hacia el valle de la Propiedad bajo la campa del Silo (Ortega et al., 2010).

Este eje continúa en cueva Peluda (Figs. 4 y 5), con un trazado rectilíneo de unos $110 \mathrm{~m}$ de longitud en sentido SSE-NNO, que finaliza en una paleosurgencia al valle de la Propiedad. Presenta numerosas chimeneas ascendentes, destacando la abundancia de raíces y concreciones. En el tramo final hay una galería inferior, con techos a $985 \mathrm{~m}$ de altitud, colmatada por gravas metamórficas (Fig. 7.F), que con dirección NNE se dirige hacia Elefante, bajo el que sondeos mecánicos detectaron los mismos cantos fluviales (Rosas et al., 2006).

Estas gravas presentan una potencia de unos 5$6 \mathrm{~m}$ de cantos cuarcíticos con matriz de arena gruesa y microconglomerática, localizadas tanto en el conducto principal de cueva del Silo como en el inferior de Peluda y Elefante. Por su parte, facies arenosas se documentan en las galerías laterales de cueva del Silo (Ortega, 2009), a cotas relacionadas altimétricamente con el nivel de arenas resedimentadas en la base de Sima de los Huesos (Aranburu et al., 2015). Estos depósitos fluviales se sitúan a 979-985 m de altitud, a +43-49 $\mathrm{m}$ del actual lecho del Arlanzón, lo que evidencia una antigua captura directa del río vinculada por cotas a niveles fluviales de la terraza T5, a +55 m en el contacto con la sierra y distante más de un kilómetro (Ortega et al., 2013).

La cueva del Compresor, localizada al norte del valle de la Propiedad, es un enrejado de pequeños conductos subhorizontales de $475 \mathrm{~m}$ de desarrollo, con marcado control estructural y direcciones preferentes SSO-NNE y ESE-ONO (Figs. 4 y 5.B). Presentan morfologías freáticas con techos abovedados a 983$985 \mathrm{~m}$ de altitud y un claro desarrollo vertical, marcado por la presencia de abundantes chimeneas de disolución con huellas de flujos ascendentes, que alcanzan los 988-992 m s.n.m. e inciden en el origen hipogénico del karst (Klimchouk, 2009; Ortega et al., 2013). La galería Norte presenta un trazado rectilíneo SSO-NNE, mientras que el sector meridional forma un dédalo verticalizado a partir de la alineación de chimeneas ascendentes, de 10-15 m de altura y cúpulas a 985 m s.n.m. La base del mayor de estos pozoschimeneas representa el punto más bajo de todo el karst, a 969 m de altitud, y conserva en sus paredes marcas de al menos una moderna inundación. Los obreros de la cantera del Compresor se referían a una inundación que llegó a anegar la parte inferior de la cantera en los años cincuenta.

Entre los conductos seccionados en Trinchera destacan las chimeneas ascendentes de Penal inferior y el conjunto final TR24-TR30 (Figs. 4 y 5.B), por representar una posible red vinculada con la descarga de aguas subterráneas en la confluencia con el valle de Valhondo (Ortega, 2009).

\section{Principales yacimientos arqueológicos}

Los yacimientos arqueológicos de Sima del Elefante, Gran Dolina y Galería muestran la ocupación de los sectores de entrada de las cavidades del nivel intermedio de Trinchera, mientras que Sima de los Huesos es un yacimiento situado en la zona oscura de Cueva Mayor (Ortega et al., 2014) (Figs. 4, 5 y 8-10). Estos yacimientos contienen un registro del Pleistoceno inferior y medio único, que ofrece la posibilidad de analizar la evolución de la ocupación humana de este enclave.

\section{El yacimiento de Sima del Elefante}

El yacimiento se ubica en la antigua paleosurgencia del nivel intermedio de Cueva Mayor y muestra una secuencia estratigráfica de unos $25 \mathrm{~m}$ de potencia, dividida en 21 unidades litoestratigráficas y tres fases sedimentarias (Carbonell et al., 2008) (Figs. 4, 5 y 8.A). 

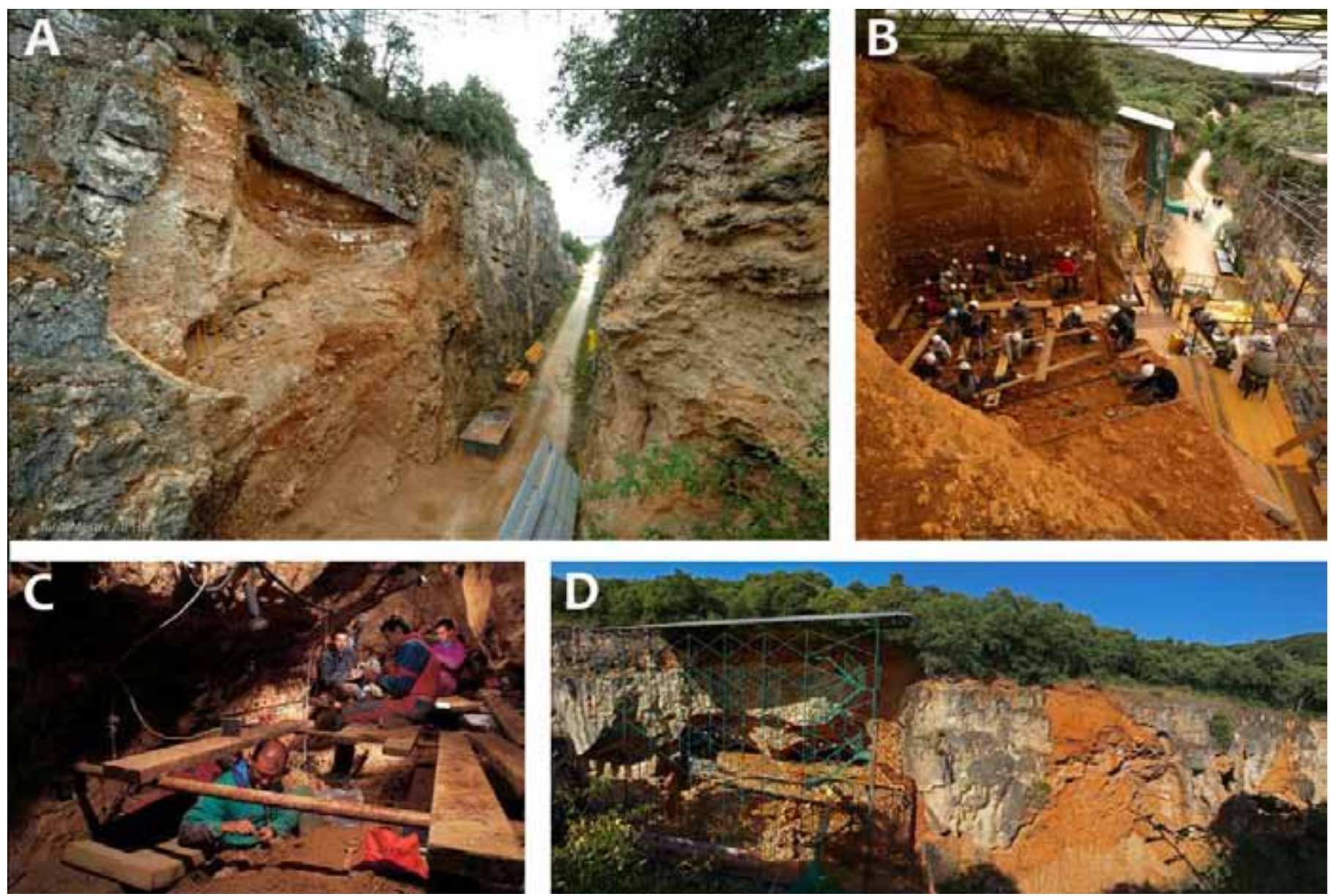

Figura 8. Yacimientos arqueológicos: A) Sima del Elefante, B) Gran Dolina, C) Sima de los Huesos, D) Galería (Fotos: A) J. Mestre-IPHES, B and D) M.A. Martín, GEE, C) J. Trueba).

Figure 8. Archaeological sites: A) Sima del Elefante, B) Gran Dolina, C) Sima de los Huesos, D) Galería (Photos: A) J. Mestre-IPHES, B and D) M.A. Martín, GEE, C) J. Trueba).

La Unidad Roja inferior (TE7-TE14) presenta una interesante asociación faunística de micromamíferos, lagomorfos, aves y castores, junto a animales de talla mediana y grande, que indican una edad preJaramillo (Cuenca-Bescós et al., 2010, 2013; Rodríguez et al., 2011). En el nivel TE9c se recuperaron restos fósiles de Homo sp. junto a huesos con marcas de antropización y objetos líticos del Modo 1, datados en 1,22 $\pm 0,16 \mathrm{Ma}$ (Carbonell et al., 2008; Bermúdez de Castro et al., 2011).

La fase media está formada por las unidades TE15TE19, en donde los niveles TE15-TE17 muestran una entrada masiva de depósitos miocenos del cercano valle de la Propiedad, detectando el límite MatuyamaBrunhes entre TE16 y TE17, con edades de luminiscentia de entre $804 \pm 47 / 864 \pm 20$ ka para TE16 y $724 \pm 20$ y $781 \pm 20$ ka para TE17 (Parés et al., 2006; Arnold et al., 2014a). Una costra de TE18 ha proporcionado dataciones de $255 \pm 12$ y $307 \pm 20$ ka y (Bischoff com. pers. en
Huguet, 2007) que sitúan TE9 en la segunda mitad del Pleistoceno medio. Este nivel presenta un amplio conjunto de grandes mamíferos, Ursus deningeri y Crocuta crocuta, asociados con útiles adscritos a Modo 2 (Lombera-Hermida, et al., 2015). La última fase sedimentaria (TE20-TE21) corresponde a la colmatación de la sección en el Pleistoceno superior.

\section{El yacimiento de Gran Dolina}

Gran Dolina, junto con Penal, representa al sector de entrada de cueva, al norte del valle de la Propiedad. Muestra una secuencia sedimentaria de $18 \mathrm{~m}$ de altura (más otros $8 \mathrm{~m}$ sondeados en su base), dividida en 11 unidades (Parés y Pérez-González, 1995) (Figs. 4-5, 8.B y 10.B).TD1 yTD2 registran un ambiente de cueva cerrada durante una fase de polaridad inversa, con un pequeño intervalo normal relacionado con el subcron 
Jaramillo o Cobb Mountain (Pérez-González et al., 2001).

La secuencia continúa con facies alóctonas que representan la apertura al exterior de este sector de la cavidad. Se caracterizan por flujos de depósitos de gravedad, en donde las unidades TD3-4 a TD6 se corresponden con fases del Pleistoceno inferior con evidencias de actividad humana relacionadas con industrias líticas del Modo 1 junto a la presencia de carnívoros y herbívoros. Actividades que se incrementan en la unidad TD6, documentando en TD6-2 comportamientos complejos, como la evidencia más antigua de canibalismo sobre unos 160 fósiles pertenecientes a unos 11 individuos de la especie Homo antecessor, con una cronología de unos 800-900 ka (Bermúdez de Castro et al., 1997; Carbonell et al., 2010; Parés et al., 2013). Estas ocupaciones presentan un importante conjunto de industrias líticas de tecnologías primitivas del Modo 1 y una rica asociación faunística típica del final del Pleistoceno inferior (Rodríguez et al., 2011; Ollé et al., 2013).

Los datos paleomagnéticos muestran el límite Matuyama-Brunhes en la parte superior del depósito fluvial de TD7 (Parés et al., 2013). TD8 representa una serie de coladas de cantos que colmatan parte de la sección y constituye un nivel exclusivamente paleontológico (Rodríguez-Gómez et al., 2014). Este nivel presenta una edad ponderada de $602 \mathrm{ka}$ y aparece sellado por un espeleotema fechado por series de uranio entre 400 y 450 ka (Falguères et al., 2001, 2013). El nivel TD9 es un nivel de limos y guano, formado en condiciones de cueva cerrada, datado porTL en $480 \pm 130 \mathrm{ka}$ (Berger et al., 2008). TD10 muestra la última fase de ocupación humana, recientemente datada por ESR entre 350-450 ka (Moreno et al., 2015), asociada a faunas del Pleistoceno Medio (Rodríguez et al., 2011; Ollé et al., 2013). Destaca la sub-unidad TD10.2 por documentar un proceso de caza y explotación especializada en bisontes, evidenciandoTD10.1 una mayor variedad de presas junto con un conjunto de industria lítica que marca una evolución local del Modo 2 al Modo 3 entre unos 430 a 250 ka (Blasco et al., 2013; García-Medrano et al., 2015). La sección de Gran Dolina se colmató con el depósito de TD11, sin restos arqueológicos, hace unos $240 \pm 44 \mathrm{ka}$, con formación de terra rosa en las grietas del techo hace $55 \pm 14$ ka (Berger et al., 2008).

\section{El yacimiento de Galería}

Al igual que los yacimientos anteriores, Galería representa la entrada de una cavidad colmatada por sedimentos, situada a unos $50 \mathrm{~m}$ de Gran Dolina (Figs. 4,
5, 8.D y 10.C). Presenta una secuencia estratigráfica con 5 unidades, identificadas como Gl a GV (PérezGonzález et al., 2001). La unidad GI está relacionada con sedimentos autóctonos depositados en condiciones de cavidad cerrada, sin restos arqueo-paleontológicos. En su tercio superior se detectó el límite Matuyama-Brunhes.

Las unidades GII a GIV son facies alóctonas y se componen de gravas fluviales carbonatadas y coladas, que lateralmente pasan a limos laminados hacia el norte. La presencia humana se ha confirmado en las Unidades GIl y GIII, representadas por un rico conjunto lítico del Modo 2, asociado con un importante registro paleontológico y dos restos fósiles humanos del Pleistoceno medio (Carbonell et al., 1999a). La cronología de este yacimiento muestra cierta disparidad, con fechas que inciden en la parte central de este periodo centradas entre 350-250 ka (Berger et al., 2008; Falguères et al., 2013; Demuro et al., 2014). La unidad Gll muestra la industria Achelense más antigua de Atapuerca, (García-Medrano et al., 2015) y en GIII, nivel TG11, se ha identificado una secuencia de 12 suelos de ocupación, formados por la alternancia de arcillas y gravas con restos arqueo-paleontológicos, que evidencia esporádicos usos de la cavidad para el aprovechamiento de los recursos cárnicos (Lorenzo y Carbonell, 1999; Cáceres et al., 2010). La torca de TN actuó como una trampa natural para los herbívoros, presentando sus carcasas un acceso diferencial entre los cánidos y los humanos.

Las Unidades GIV y GV colmatan la cueva, documentando la formación del espeleotema a techo de GIV entre hace 160 y $210 \mathrm{ka}$, por Series de Uranio y ESR (Falguères et al., 2013).

\section{El yacimiento de Sima de los Huesos}

Sima de los Huesos se encuentra en la zona oscura de la cavidad, en la base de un pozo de unos $13 \mathrm{~m}$ de profundidad y a unos 500 de la actual entrada de Cueva Mayor (Figs. 4, 5, 8.C y 10.D). Presenta una secuencia estratigráfica con cinco fases sedimentarias, que incluyen 12 unidades litoestratigráficas (LU) (Arsuaga et al., 2014). La secuencia se inicia con la LU1, compuesta por margas miocenas resedimentadas identificadas con un proceso de disolución y colapso. Sobre estas margas se desarrolla la LU2, una costra estalagmítica situada al sur de la vertical del pozo. La LU3 está compuesta por arenas y limos laminados, resedimentadas de la sala de los Cíclopes (Aranburu et al., 2015) de origen fluvial y polaridad inversa (Arnold et al., 2014b).

La LU5 representa el nivel de arcillas rojas estéri- 
les (Bischoff et al., 1997) con indicios de polaridad inversa (Arnold et al., 2014b), dando polaridad normal LU6. Esta unidad contiene la muestra más representativa de los homininos del Pleistoceno medio, con restos de 28 individuos de hace unos $430 \mathrm{ka}$ (Arsuaga et al., 2014). La excepcionalidad del depósito apunta a una acumulación de origen antrópico, y el hallazgo de un único bifaz sugiere la evidencia un ritual simbólico. EI nivel LU7 es una brecha de barro fosilífera con restos de más de un centenar de osos (Ursus deningeri) junto a carnívoros del Pleistoceno medio. Una colada estalagmítica (LU10) sella la secuencia, depositándose sobre ella una capa de guano (LU11).

\section{Espeleogénesis y ocupación humana del medio kárstico}

Los grandes conductos horizontales, como los documentados en este enclave, se asocian a periodos de relativa estabilidad del nivel de base y una serie escalonada de conductos es el resultado de la evolución geodinámica regional, estableciendo una relación de dependencia con el encajamiento y reorganización hídrica local (Ford y Williams, 2007; Palmer, 2007). La evolución del karst de la Sierra de Atapuerca sugiere escasas evidencias de karstificación pre-cuaternaria, mostrando únicamente una relación altimétrica entre la Superficie de Erosión SE2 (Mioceno medio) y el pequeño conjunto de La Rasa (cueva Ciega-cueva Paredeja) (Ortega, 2009), en contraposición con el control que ejercerá la dinámica fluvial del Arlanzón en relación con las resurgencias al río Pico.

El término de Torcas concentra un sistema multinivel con tres niveles principales escalonados de conductos sub-horizontales, desarrollados a partir del encajamiento del valle medio del Arlanzón, presentando surgencias activas que drenan al valle de Valhondo durante la formación de cada nivel fluvial (T2-T5), cuyo descenso da paso a fases vadosas (Fig. 9). En las fases de inactividad, los grandes portalones serán utilizados por los humanos y los carnívoros, hasta su colmatación (Fig. 8).

En este esquema espeleogenético el nivel superior está relacionado con los niveles de base, próximos entre sí, de la superficie SE4 y terrazaT2 (Ortega et al., 2013), favoreciendo la ampliación de un gran conducto paragenético, y la incisión inicial de Valhondo y del valle del Pico. Los datos paleomagnéticos muestran una fase de polaridad inversa para la deposición de los sedimentos arcillosos y la costra que los sella, relacionada con el cron Matuyama (Parés et al., in revision). Fase que encuadra con el espacio temporal de entre 2,2 y 1,14-1,22 Ma propuesto para la dura-

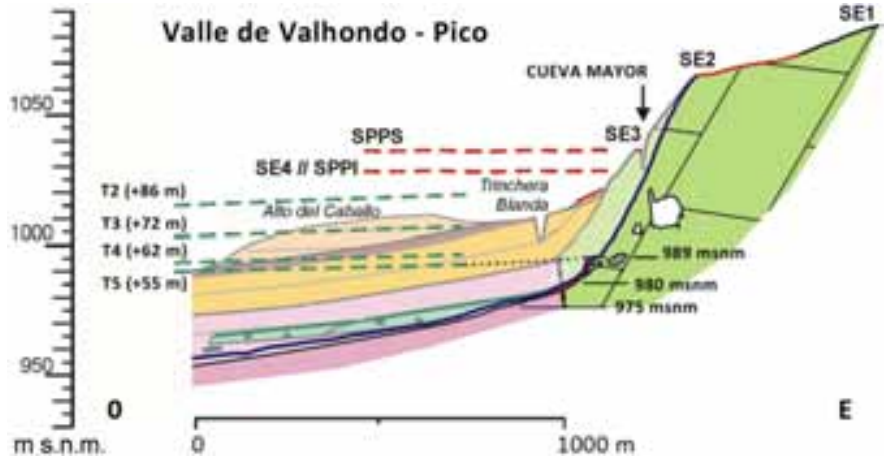

Figura 9. Sección transversal del valle de Valhondo, mostrando las relaciones entre los niveles kársticos y el relieve desarrollado durante el Neógeno y el Cuaternario (basado en Ortega, 2009). Figure 9. Cross-section of the Valhondo valley, indicating the relationship between the cave levels and the landforms developed during the Neogene and Quaternary (modified from Ortega, 2009).

ción de estos niveles de base (Benito-Calvo et al., en este volumen).

La evolución del nivel de base en relación con la incisión fluvial, vinculada a la terrazaT3, trajo consigo el desarrollo de morfologías vadosas, con secciones mixtas tipo "ojo de cerradura" y encajamientos que favorecen el aumento de los volúmenes en los puntos de conexión con el nivel intermedio, como el del salón del Coro (Ortega et al., 2013). El abandono por las aguas de su más antigua y elevada paleosurgencia, en el final de la galería de las Estatuas, implicaría una primigenia fase de utilización por los primeros homininos y carnívoros durante el inicio del Pleistoceno inferior, aunque una potente estratigrafía sella el acceso a estos posibles depósitos.

Con la estabilidad del nuevo nivel de base de laT3 (+72 m en la sierra) se relaciona la formación del nivel intermedio y sus paleosurgencias de Elefante $y$ Dolina (Ortega, 2009). Los sedimentos arcillosos, vinculados con el desarrollo de este nivel en Cueva Mayor, presentan paleomagnetismo negativo relacionado con el cron Matuyama (Parés et al., 2010, in revision), apoyando la cronología del nivel y la terraza T3, cuya edad estimada es de $1,14 \pm 0,13 \mathrm{Ma}$ (Moreno et al., 2012).

Por su parte, la cronología de la secuencia estratigráfica de la Unidad Roja Inferior de Elefante ha proporcionado una edad mínima de 1,13-1,2 Ma y polaridad inversa (Carbonell et al., 2008). Esta fecha representa la primera entrada conocida en la actualidad tanto de los homininos y animales como de sedimentos al sector paleosurgente, durante un período cálido y húmedo, coincidiendo con la primera llegada de los homininos al sur de Europa (Fig. 10.A inferior). La posición original de esta unidad junto a las evi- 


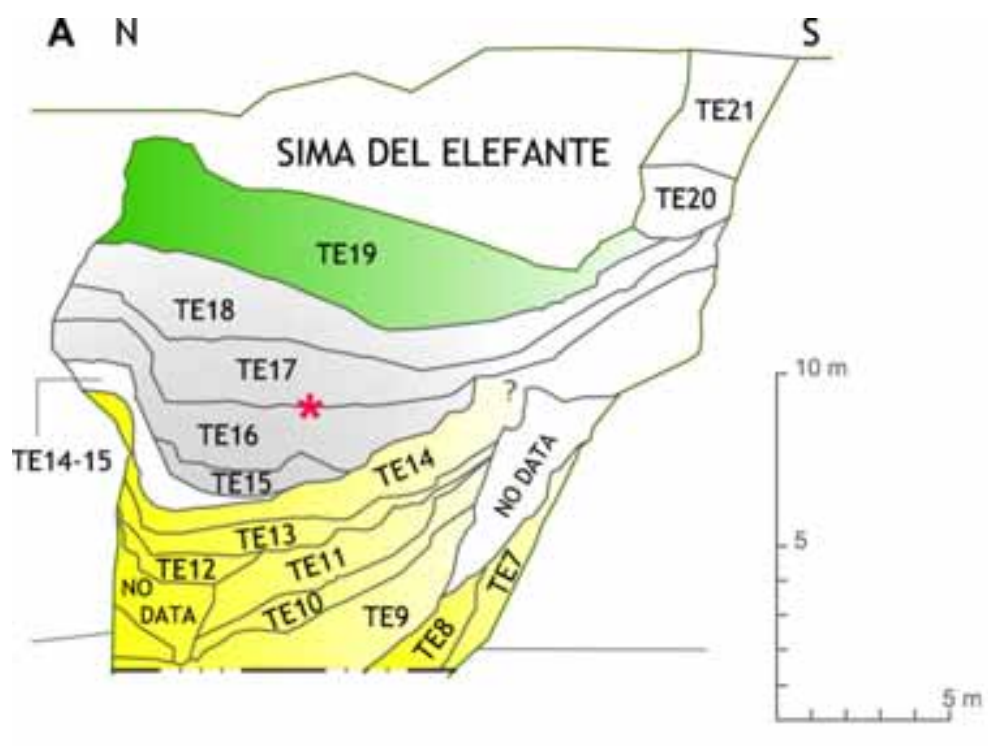

B N

S
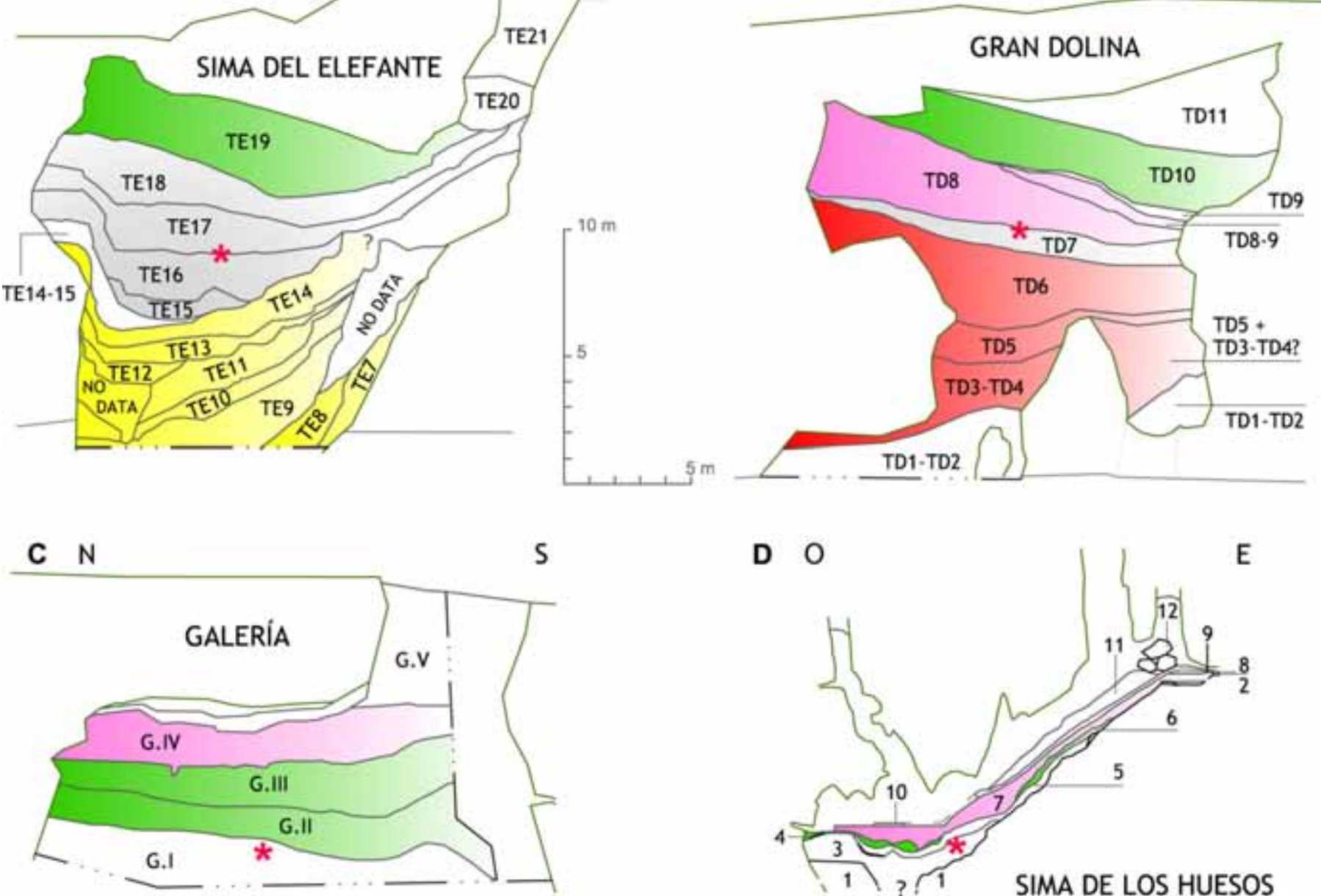

Figura 10. Secuencias estratigráficas que indican la evolución de las diferentes fases de ocupación humana en las Cuevas de Atapuerca durante el Pleistocene inferior y medio. A) Sima del Elefante (basado en Rosas et al., 2006). B) Gran Dolina (basado en Pérez-González et al., 2001). C) Galería (basado en Ortega, 2009). E) Sima de los Huesos (basado en Aranburu et al., 2014). Leyenda: Amarillo: Primeros asentamientos humanos, Periodo pre-Jaramillo. Rojo: Segunda fase de ocupación humana, final del Pleistoceno inferior; Verde:Tercera fase de asentamientos humanos, Pleisoceno medio medio; Morado: Uso exclusivo por animales salvajes; Gris: depósitos sedimentarios de alta energía, entrada de cuevas. El asterisco marca la posición del límite Matuyama-Brunhes.

Figure 10. Stratigraphic sequences showing the evolution of the different phases of human occupation in the Atapuerca caves during the Early-Middle Pleistocene. A) Sima del Elefante (based on Rosas et al., 2006), B) Gran Dolina (based on Pérez-González et al., 2001). C) Galería (based on Ortega, 2009). E) Sima de los Huesos (based on Aranburu et al., 2014). Legend: yellow: first human occupation, PreJaramillo Period. Red: second human occupation, lower Early Pleistocene. Green: third human occupation, mid-Middle Pleistocene. Purple: wildlife occupation; grey: high-energy sedimentary deposits at the cave entrances. The asterisk marks the position of the Matuyama-Brunhes boundary.

dencias acuáticas de su formación (Rodríguez et al., 2011), sugieren una edad de enterramiento próxima o algo posterior al abandono de laT3 del Arlanzón.

El nivel de base de la T4 del Arlanzón (+64 m en la sierra), con una edad ponderada de 0,85 Ma y polaridad inversa, provocó la formación en la zona inundada del conducto de Galería y el encajamiento vadoso de los tramos distales del nivel intermedio (Cíclopes, Elefante y Dolina) desarrollándose cañones y anchas secciones en "ojo de cerradura" (Ortega et al., 2012).

La evolución de este nivel de base conllevó el colapso de techos y de los sedimentos que contenían. Gran Dolina muestra como, tras la acumulación de grandes bloques de colapso (TD2), se produce la apertura de este sector de la cueva, dando paso al inicio de una nueva fase de sedimentación alóctona (Fig. 10.B inferior), relacionada con la llegada de Homo antecessor en torno a 1,0 Ma, posiblemente durante el MIS 25 (Carbonell et al., 1999b; Vallverdú, et al., 2001; Parés et al., 2013). Durante esta fase la cueva debió presentar una rampa descendente, desde la sección de Penal, según las relaciones fau- 
nísticas de Penal y Dolina (Fernández-Jalvo, 1995; Made, 2013).

Dolina y Elefante documentan fases de recarga por avenidas torrenciales que se sumen por las cuevas (Figs. 9.A y 9.B centro). Las facies aluviales de TD7 de Dolina y TE16-17 en Elefante marcan ambientes Iluviosos relacionados con el límite Matuyama-Brunhes (Parés et al., 2006, 2013). Este límite aparece registrado en los sedimentos del nivel GI de Galería (PérezGonzález et al., 2001).

La progresiva incisión que imponen las terrazas T5 (+55 m) y T6 (+46 m) del Arlanzón, posicionadas en el contacto con la sierra a $991 \mathrm{~m}$ y $982 \mathrm{~m}$ de altitud respectivamente, desarrolló el nivel inferior a partir de un nuevo reajuste del flujo hacia el oeste, que marcará nuevos puntos surgentes en Valhondo (Fig. 9) (Ortega, 2009). En este nivel se localizan los depósitos fluviales de cueva del Silo y el conducto inferior de Peluda-Elefante, relacionados con el nivel de base de la T5 del Arlanzón, primera terraza con polaridad normal (Benito-Calvo et al., 2008) y una edad ponderada de 0,66 Ma (Moreno et al., 2012; Benito-Calvo et al., en este volumen).

La pérdida directa del Arlanzón impulsó el propio desarrollo del nivel inferior, con la disolución de sus conductos, junto a importantes colapsos de los techos que permiten la entrada de las margas miocenas en varios puntos de cueva del Silo y Sima de los Huesos (Ortega et al., 2013). El colapso y deformación de las unidades sedimentarias situadas en la entrada de Elefante (TE7 a TE17) puede estar relacionado con este evento, así como la excavación y relleno que afecta a los niveles TD5-TD8 de Dolina (PérezGonzález et al., 2001; Canals et al., 2003). Estos procesos se relacionan con las unidades TE17 y TD8, así como con los limos que sellan las gravas metamórficas del cueva del Silo, que marcan el inicio de la polaridad normal (Parés et al., 2006, 2013, in revision), confirmando una edad mesopleistocena para la entrada y deposición de los depósitos fluviales, así como para los procesos de deformación y colapso.

Todo esto muestra una importante actividad hídrica durante la fase inicial del Pleistoceno medio, que sugiere cavidades inseguras e inadecuadas para su uso. El registro arqueológico representado por los niveles TD8 de Dolina y TE17 de Elefante constata la ausencia de actividad humana (Ollé et al., 2013), enmarcada dentro de un relativo vacío poblacional del continente europeo entre $0,7-0,5 \mathrm{Ma}$, planteando algunos autores la existencia de condicionantes climáticos o medioambientales a este despoblamiento. A este respecto, TD8 muestra el uso exclusivo como guarida y refugio temporal de hienas, lobos, linces, jaguares y osos (Blasco et al., 2011) (Fig. 10.A). Para estas fases, las dinámicas sedimentarias llegan prácticamente a colmatar las entradas, creando en Dolina un ambiente cerrado de cueva que facilitó la precipitación de un espeleotema, entre 0,40-0,45 Ma (Falguères et al., 2013), y la posterior acumulación del nivel de guano TD9. Condiciones de un entorno hostil, en donde existe mayor competencia con los carnívoros y un paisaje más homogéneo con alto predominio de taxones mediterráneos (Rodríguez-Gómez et al., 2014).

Una nueva fase en la evolución del karst está marcada por procesos de colapso y reaperturas de cavidades, que serán utilizadas por los nuevos grupos de inmigrantes achelenses que ocuparon toda Europa hace 0,5 Ma (Dennel et al., 2011; Mosquera et al., 2013) y que en Atapuerca realizarán un uso sistemático del paisaje kárstico (fig. 10) (Ortega et al., 2014). A esta fase corresponden los colapsos del techo de Galería que originaron Tres Simas y Covacha Zarpazos y aportaron acceso y luminosidad a su interior. Esta cavidad presenta un uso reiterado y esporádico de los homininos, en competencia con los carnívoros, por las carcasas de los herbívoros que caían por las torcas (GII-III, Fig. 10.C), documentando las fases más antiguas del Achelense de toda Atapuerca (Cáceres et al., 2010; García-Medrano et al., 2015).

Un nuevo colapso del techo de Gran Dolina facilitó de nuevo el acceso a su interior (Mallol y Carbonell, 2008), documentando en el nivel TD10 la ocupación más intensa del registro de Trinchera (Fig. 10. $\mathrm{B}$ superior), en torno a $0,40-0,35 \mathrm{Ma}$, con asentamientos tipo campamento base y tecnologías de transición del Modo 2 al 3 (TD10.1 y 2) (Ollé et al., 2013; García-Medrano et al., 2015). Por último, el nivel TE19 de Elefante colmata la secuencia con ocupaciones humanas de la segunda mitad del Pleistoceno medio (Fig. 10.A superior) (Carbonell et al., 2014).

Un colapso de la bóveda, en el lateral de la sala de los Cíclopes, propició el acceso al entorno de Sima de los Huesos tanto a los osos como a los homininos. Este yacimiento, con una cronología de $0,43 \mathrm{Ma}$, conserva un importante conjunto de fósiles humanos (Fig. 10.D), en donde su ubicación, intencionalidad de la acumulación y presencia de un único bifaz achelense sugieren la existencia de complejidad y simbolismo (Arsuaga et al., 2014).

Todas estas evidencias revelan la intensidad de la ocupación del territorio durante el Pleistoceno medio. El uso diferencial de los espacios para el desarrollo de distintas actividades sugiere una exploración, planificación y control del paisaje kárstico, transformándolo en un paisaje social con la intención de apropiarse de la zona oscura de la cueva en el yacimiento de Sima de los Huesos. 
Con el nivel de la terraza T7 ( $+40 \mathrm{~m}$ en la sierra) se relaciona la última interacción entre los niveles de base del Arlanzón y del Pico, a través de los puntos bajos de cueva del Silo, Peluda y Compresor. El descenso del nivel de base de la terraza T8 $(+38 \mathrm{~m})$, con edades por ESR de 0,37 y 0,40 Ma (Moreno et al., 2012), representa la desconexión hidrológica del karst, con resurgencias inactivas y colgadas, convirtiendo Valhondo y la cabecera del Pico en un valle relicto.

Esta nueva etapa se caracteriza por la fosilización de los sectores de entrada, imposibilitando el acceso a su interior a desde los inicios del Pleistoceno superior. Los retrocesos de ladera y nuevos colapsos de bóvedas próximas a la superficie permitirán el acceso a la antigua y más elevada paleosurgencia de la galería de las Estatuas de Cueva Mayor (Ortega, 2009), en donde recientes excavaciones han descubierto ocupaciones del Paleolítico medio, sobre una compleja secuencia sedimentaria que tuvo que iniciarse en las fases iniciales del Pleistoceno inferior. Este registro se complementa con asentamientos al aire libre del Paleolítico medio, entre los que destacan los yacimientos de Hundidero y $\mathrm{HC}$ en el Valle del Pico, datados entre 71-48 ka (Navazo, 2006; Arnold et al., 2013). Las evidencias del Paleolítico superior son escasas, destacando los yacimientos de Orquídeas (Mosquera et al., 2007).

Durante el Holoceno, en la Prehistoria reciente, se inicia una cuarta fase de ocupación del karst caracterizada por el uso sistemático de todas las cavidades de la Sierra de Atapuerca, destacando el carácter simbólico y sagrado que imprimieron a este espacio. Durante los albores de la Alta Edad Media se erige en lo alto de la Sierra de Atapuerca la iglesia de San Vicente, cuya advocación daría nombre al sector meridional de la misma, bajo el que se desarrollan las cavidades, cuyo espacio se protegerá con un territorio comunal (Ortega et al., 2012).

\section{Conclusiones}

La Sierra de Atapuerca contiene un sistema multinivel de cavidades de conductos subhorizontales de origen paragenético y evolución vadosa, con direcciones de drenaje S-N desarrolladas en paralelo a la ladera y al eje anticlinal. Las diferentes paleosurgencias 0 manantiales de los valles de la Propiedad y Valhondo aportarán, durante el Pleistoceno, un importante caudal a la cabecera del río Pico. Los conductos aparecen escalonados en tres niveles principales, en coincidencia altimétrica con los niveles de base que alcanzaron en el contacto con la sierra las terrazas fluviales T2
(+86 m), T3 (+72 m) y laT5 (+55 m). La incisión fluvial produjo la progresiva liberación de las aguas de los conductos superiores, así como la formación progresiva de los niveles más bajos del karst. Las cuevas accesibles fueron utilizadas por la fauna y los homininos, conservando un registro arqueo-paleontológico desde hace más de 1,2 Ma hasta el final del Pleistoceno Medio, documentado tres fases culturales de ocupación humana en los diferentes yacimientos de Trinchera (Sima del Elefante, Gran Dolina y Galería) y Sima de los Huesos (Cueva Mayor).

Este importante registro no representa una ocupación continua sino que muestra significativas ausencias que apoyan la existencia de discontinuidades en el poblamiento europeo durante el Pleistoceno inferior y medio. La primera fase está representada por la llegada de los primeros homininos a Europa, documentada en Sima del Elefante, con ocupaciones esporádicas pre-Jaramillo de hace 1,2 Ma, poco después del abandono del nivel de base de la terraza T3 del Arlanzón.

La segunda fase está relacionada con los asentamientos de Homo antecessor, registrados enTD4-TD6 de Gran Dolina, caracterizados por una ocupación reiterada al final del Pleistoceno inferior.

La tercera fase se caracteriza por una nueva llegada de poblaciones con culturas líticas achelenses, que van a presentar una sistemática e intensa ocupación de este espacio entre hace 0,45 y $0,25 \mathrm{Ma}$, con una diversidad de actividades desarrolladas en las diferentes cavidades, en donde destacan los yacimientos de Galería y TD10 de Dolina, así como de Sima de los Huesos, por albergar un complejo y completo registro de las actividades de los homininos europeos del Pleistoceno medio.

La colmatación por sedimentos de las entradas de las cuevas es generalizada en el Pleistoceno superior, aunque nuevos colapsos han posibilitado el acceso a otras cavidades, como es el caso de la galería de las Estatuas o el Portalón de Cueva Mayor. Finalmente, durante el Holoceno, se generaliza la ocupación del karst en las fases de la Prehistoria reciente.

\section{Agradecimientos}

Este trabajo ha sido financiado por los proyectos del MINECO CGL 2009-12703-C03-01, CGL2012-38434C03-02, y las excavaciones han sido financiadas por la Junta de Castilla y León. Los autores quieren agradecer al Equipo de Investigación de Atapuerca (EIA) y al Grupo Espeleológico Edelweiss (GEE) el apoyo científico y logístico. A.I.O.M. tiene una Beca de investigación Posdoctoral de la Fundación Atapuerca. 


\section{Referencias}

Aranburu, A., Arsuaga, J.L. and Sala, N. 2015. The stratigraphy of the Sima de los Huesos (Atapuerca, Spain) and implications for the origin of the fossil hominin accumulation. Quaternatiy International. doi: 10.1016/j.quaint.2015.02.044.

Arnold, L.J., Demuro, M., Parés, J.M., Pérez-González, A., Arsuaga, J.L., Bermúdez de Castro, J.M. and Carbonell, E., 2014a. Evaluating the suitability of extended-range luminescence dating techniques over early and Middle Pleistocene timescales: published datasets and case studies from Atapuerca, Spain. Quaternary International. http://dx.doi.org/10.1016/j.quaint.2014.08.010.

Arnold, L.J., Demuro, M., Parés, J.M., Arsuaga, J.L., Aranburu, A., Bermúdez de Castro, J.M. and Carbonell, E. 2014b. Luminescence dating and palaeomagnetic age constraint on hominins from Sima de los Huesos, Atapuerca, Spain. Journal of Human Evolution 67, 85107.

Arnold, L.J., Demuro, M., Navazo Ruiz, M., Benito-Calvo, A. and Pérez-González, A., 2013. OSL dating of the Middle Palaeolithic Hotel California site, Sierra de Atapuerca, north-central Spain. Boreas, 42, 285-305.

Arsuaga, J.L., Martínez, I., Arnold, L.J., Aranburu, A., Gracia, A., Sharp, W.D., Quam, R.M., Falguères, C., Pantoja, A., Bischoff, J., Poza, E., Parés, J.M., Carretero, J.M., Demuro, M., Lorenzo, C., Sala, N., Martinón, M., García, N., Alcázar, A., Cuenca, G., Gómez, A., Moreno, D., Pablos, A., Shen, C.C., Rodríguez, L., Ortega, A.I., García, R., Bonmatí, A., Bermúdez de Castro, J.M. and Carbonell, E. 2014. Neandertal roots: Cranial and chronological evidence from Sima de los Huesos, Science 344, 1358-1363.

Benito-Calvo, A. 2004. Análisis geomorfológico y reconstrucción de paleopaisajes neógenos y cuaternarios en la Sierra de Atapuerca y el valle medio del río Arlanzón. Universidad Complutense de Madrid, Madrid. Tesis Doctoral.

Benito-Calvo, A. and Pérez-González, A. 2007. Erosion surfaces and Neogene landscape evolution in the NE Duero Basin (north-central Spain). Geomorphology 88, 226241.

Benito-Calvo, A., Pérez-González, A. and Parés, J.M. 2008. Quantitative reconstruction of Late Cenozoic landscape: a case study in the Sierra de Atapuerca (Burgos, Spain). Earth Surf Proc Land, 33, 196-208.

Benito-Calvo, A. and Pérez-González, A. 2015. Geomorphology of the Sierra de Atapuerca and the Middle Arlanzón Valley (Burgos, Spain). Journal of Maps, 11, 535-544.

Benito-Calvo, A., Ortega, A.l., Navazo, M., Moreno, D., Pérez-Gonzalez, A., Parés, J.M., Bermúdez de Castro, J.M. and Carbonell, E. (submitted to this volumen). Pleistocene evolution of the Arlanzón valley: implications for the formation of the endokarst system and the open air archaeological sites of the Sierra de Atapuerca (Burgos, España). Boletín Geológico y Minero.

Berger, G.W., Pérez-González, A., Carbonell, E., Arsuaga, J.L., Bermúdez de Castro, J.M. and, Ku, T.L. 2008.
Luminiscence chronology of cave sediments at the Atapuerca paleoanthropological site, Spain. Journal of Human Evolution, 55, 300-311.

Bermúdez de Castro, J.M., Arsuaga, J.L., Carbonell, E., Rosas, A., Martínez, I. and Mosquera, M. 1997. A hominid from the Lower Pleistocene of Atapuerca. Spain: possible ancestor to Neandertals and modern human. Science, 276, 1392-1395.

Bermúdez de Castro, J.M. and Martinón-Torres M. 2013. A new model for the evolution of the human Pleistocene populations of Europe. Quaternary International, 295, 102-112.

Bischoff, J.L., Fiztpatrick, J.A., León, L., Arsuaga, J.L., Falguères, C., Bahain, J.J. and Bullen, T. 1997. Geology and preliminary dating of the hominid-bearing sedimentary fill of the Sima de los Huesos Chamber, Cueva Mayor of the Sierra de Atapuerca, Burgos, Spain. Journal of Human Evolution, 33, 129-154.

Blasco, R., Rosell, J., Made, J.v.d., Rodríguez, J., Campeny, G., Arsuaga, J.L., Bermúdez de Castro, J.M. and Carbonell, E. 2011. Hiding to eat: the role of carnivores in the early Middle Pleistocene from the TD8 level of Gran Dolina (Sierra de Atapuerca, Burgos, Spain). Journal of Archaeological Science, 38, 3373-3386.

Blasco, R., Rosell, J., Fernández-Peris, J., Arsuaga, J.L., Bermúdez de Castro, J.M and., Carbonell, E. 2013. Environmental availability, behavioural diversity and diet: a zooarchaeological approach from the TD10-1 sublevel of Gran Dolina (Sierra de Atapuerca, Burgos, Spain) and Bolomor Cave (Valencia, Spain). Quaternary Science Reviews, 70, 124-144.

Brugal, J.Ph., Diez-Lomana, C., Huguet. R, Michel, P. and Rosell, J. 2006. Karstic cavities, natural bone accumulations and discrete human activities in the european palaeolithic : some case studies. In (Haws, J.A., Hockett, B.S. \& Brugal, J.P., eds.) Palaelithic Zooarchaeology in Practice. BAR international Series, 1564, Oxford, pp. 117.

Cáceres, I., Huguet, R., Rosell, J., Esteban, M., Saladié, P., Díez, J.C., Ollé, A., Vallverdú, J., García, P. and Carbonell, E. 2010. El yacimiento de Galería (Sierra deAtapuerca, Burgos, España): un enclave para la obtención de recursos cárnicos en el Pleistoceno Medio. In: Baquedano, E. and Rosell, J. (eds.), $1^{a}$ Reunión de científicos sobre cubiles de hiena (y otros grandes carnívoros) en los yacimientos arqueológicos de la Península Ibérica. Zona Arqueológica, 13, 157-165.

Canals, A., Vallverdú, J. and Carbonell, E. 2003. TD6 Level in Relation to Homo antecessor (Lower Pleistocene) at the Site of Atapuerca, North-Central Spain. Geoarchaeology, 18, 481-504.

Carbonell Roura, E., Rosas González, A. and Díez FernándezLomana, J. C. (Eds) 1999a. Atapuerca: Ocupaciones humanas y paleoecología del yacimiento de Galería, Arqueología en Castilla y León, 7. Ed. Junta de Castilla y León. Zamora. 390 pp.

Carbonell, E., Mosquera, M., Rodríguez, X.P. and Sala, R. 1999b. Out of Africa: the dispersals of the earliest technical systems reconsidered. Journal of Anthropological Archaeology, 18, 119-136. 
Carbonell, E., Bermúdez de Castro, J.M., Parés, J.M., PérezGonzález, A., Cuenca-Bescós, G., Ollé, A., Mosquera, M., Huguet, R., Made, J.v.d., Rosas, A., Sala, R., Vallverdú, J., García, N., Granger, D.E., Martinón, M., Rodríguez, X.P., Stock, G.M., Vergès, J.M., Allue, E., Burjachs, F., Cáceres, I., Canals, A., Benito, A., Díez, C., Lozano, M., Mateos, A., Navazo, M., Rodríguez, J., Rosell, J. and Arsuaga, J.L. 2008. The first hominin of Europe. Nature, 425, 465-470.

Carbonell, E., Cáceres, I., Lozano, M., Saladié, P., Rosell, J., Lorenzo, C., Vallverdú, J., Huguet, R., Canals, A. and Bermúdez de Castro, J.M. 2010. Cultural cannibalism as a paleoeconomic system in the European Lower Pleistocene. Current Anthropology, 51, 539-549.

Cuenca-Bescós, G., Rofes, J., López-García, J.M., Blain, H.A., de Marfá, R.J., Galindo, M.A, Bennásar, L.M., Melero, J.M., Arsuaga, J.L., Carbonell, E. and Bermúdez de Castro, J.M. 2010. Biochronology of Spanish Quaternary small vertebrate faunas. Quaternary International, 212, 109-119.

Cuenca-Bescós, G., Rofes, J., López-García, J.M., Blain, H.A., Rabal, R., Sauqué, V., Arsuaga, J.L., Bermúdez de Castro, J.M. and Carbonell, E. 2013. The small mammals of Sima del Elefante (Atapuerca, Spain) and the first entrance of Homo in Western Europe. Quaternary International, 295, 28-35.

Demuro, M., Arnold, L.J., Parés, J.M., Pérez-González, A., Ortega, A.I., Arsuaga, J.L., Bermúdez de Castro, J.M. and Carbonell, E. 2014. New Luminescence Ages for the Galería Complex Archaelogical Site: Resolving Chronological Uncertainties on the Acheulean Record of the Sierra de Atapuerca, Northern Spain, Plos One, 9(10), e110169.

Dennell, R.W., Martinón-Torres, M. and Bermúdez de Castro, J.M. 2011. Hominin variability, climatic instability and population demography in Middle Pleistocene Europe. Quaternary Science Reviews, 30, 1511-1524.

Eraso, A., Domínguez, M.C., Pérez González, A., Martín Merino, M.A. and Ortega, A.I. 1998. Estimación de las paleovelocidades y sentidos de circulación del agua en el karst donde se emplaza el yacimiento arqueológico de la Sierra de Atapuerca (Burgos). Geogaceta, 23, 3942.

Falguères, C., Bahain, J.J., Yokohama, Y., Bischoff, J.L., Arsuaga, J.L., Bermúdez de Castro, J.M., Carbonell, E. and Dolo, J.M. 2001. Datation par RPE et U-Th des sites pléistocènes d'Atapuerca: Sima de los Huesos, Trinchera Dolina et Trinchera Galería. Bilan géochronologique. L'anthropologie, 105, 71-81.

Falguères, C., Bahain, J.J., Bischoff, J.L., Pérez-González, A., Ortega, A.I., Ollé, A., Quiles, A., Ghaleb, B., Moreno, D., Dolo, J.M., Shao, Q., Vallverdú, J., Carbonell, E., Bermúdez de Castro, J.M. and Arsuaga, J.L. 2013. Combined ESR/U-Series chronology of Acheulian Hominid-bearing layers at Trinchera Galería site, Atapuerca, Spain. Journal of Human Evolution, 196, 122137.

Fernández-Jalvo, Y. 1995. Small mammal taphonomy at Trinchera de Atapuerca (Burgos, Spain). A remarkable example of taphonomic criteria used for stratigraphic correlations and palaeoenvirnment interpretations.
Palaeogeography, Palaeoclimatology, Palaeoecology, 114, 167-195.

Ford, D.C. and Williams, P. 2007. Karst Hydrogeology and Geomorphology. Ed. John Wiley \& Sons Ltd. 562 pp.

García-Medrano, P., Ollé, A., Mosquera, M., Cáceres, I., Díez, C. and Carbonell, E. 2015. The nature of technological changes: The Middle Pleistocene stone tool assemblages from Galería and Gran Dolina-subunit TD10.1 (Atapuerca, Spain), Quaternary International, http://dx.doi.org/10.1016/j.quaint.2015.03.006

Huguet, R. 2007. Primeras ocupaciones humanas en la Península Ibérica: paleoeconomía en la Sierra de Atapuerca (Burgos) y en la Cuenca de Guadix-Baza (Granada) durante el Pleistoceno Inferior. Ph. D. Thesis, Universitat Rovira i Virgili, Tarragona, Spain.

Klimchouk, A. 2009. Morphogenesis of hypogenic caves. Geomorphology 106, 100-117.

Leroy, S.A.G., Arpe, K. and Mikolajewicz, U. 2011. Vegetation context and climatic limits of the Early Pleistocene hominin dispersal in Europe. Quaternary Science Reviews, 30, 1448-1463.

Lombera-Hermida, A. de, Bargalló, A., Terradillos-Bernal, M., Huguet, R., Vallverdú, J., García-Antón, M.D., Mosquera, M., Ollé, A., Sala, R., Carbonell, C. and Rodríguez-Álvarez, X.P. 2015. The lithic industry of Sima del Elefante (Atapuerca, Burgos, Spain) in the context of Early and Middle Pleistocene technology in Europe. Journal of Human Evolution, http://dx.doi.org/10.1016/j.jhevol.2015.03.002

Lorenzo, C. and Carbonell, E. 1999. Representación espacial de los suelos de ocupación del nivel TG11 de Trinchera Galería, Sierra de Atapuerca, Burgos. En: Carbonell, E., Rosas, A., Díez, J.C. (eds) Atapuerca: Ocupaciones humanas y paleoecología del yacimiento de Galería. Arqueología en Castilla y León, 7, 74-94.

MacDonald, K., Martinón-Torres, M., Dennell, R.D. and Bermúdez de Castro, J.M. 2013. Discontinuity in the record for hominin occupation in south-western Europe: Implications for occupation of the middle latitudes of Europe. Quaternary International, 271, 84-97.

Made, J. van der 2013. First description of the large mammals from the locality of Penal, and updated faunal lists for the Atapuerca ungulates - Equus altidens, Bison and human dispersal into Western Europe. Quaternary International, 295, 36-47.

Mallol, C. and Carbonell, E. 2008: The collapse of Gran Dolina cave, Sierra de Atapuerca, Spain: Site formation processes of layerTD10-1. Geoarchaeology, 23, 13-41.

Martín, M.A., Domingo, S. and Antón, T. 1981. Estudio de las cavidades de la zona BU-IV.A (Sierra de Atapuerca). Kaite, 2, 41-76.

Martín, M.A. 2000. Topografía espeleológica en Atapuerca. Cubía, 2, 40-46.

Moreno, D., Falguères, C., Pérez-González, A., Duval, M., Voinchet, P., Benito-Calvo, A., Ortega, A.l., Bahain, J.J., Sala, R., Carbonell, E., Bermúdez de Castro, J.M. and Arsuaga, J.L. 2012. ESR chronology of alluvial deposits in the Arlanzón valley (Atapuerca, Spain): Contemporaneity with Atapuerca Gran Dolina site. Quaternary Geochronology, 10, 418-423.4 
Moreno, D., Falguères, C., Pérez-González, A., Voinchet, P., Ghaled, B., Despriée, J., Bahain, J.-J., Sala, R., Carbonell, E., Bermúdez de Castro, J.M. and Arsuaga, J.L. 2015. New radiometric dates on the lowest stratigraphical section (TD1 to TD6) of Gran Dolina site (Atapuerca, Spain). Quaternary Geochronology, http://dx.doi.org/10.1016/j.quageo.2015.05.007

Mosquera, M., Ollé, A., Pérez-González, A., Rodríguez, X. P., Vaquero, M., Vergès. J. M. and Carbonell, E. 2007. Valle de las Orquídeas: un yacimiento al aire libre del Pleistoceno superior en la Sierra de Atapuerca (Burgos). Trabajos de Prehistoria, 64, 143-155.

Mosquera, M., Ollé, A. and, Rodríguez, X.P. 2013. From Atapuerca to Europe: Tracing the earliest peopling of Europe, Quaternary International, 295, 130-137.

Muttoni, G., Scardia, G., Kent, D.V., Morsiani, E., Tremolada, F., Cremaschi, M. and Peretto, C. 2011. First dated human occupation of Italy at $\sim 0.85 \mathrm{Ma}$ during the late Early Pleistocene climate transition. Earth and Planetary Science Letters, 307, 241-252.

Navazo, M. 2006. Sociedades cazadoras-recolectoras en la Sierra de Atapuerca durante el Paleolítico medio: Patrones de asentamiento y estrategias de movilidad. Ph.D. thesis, Universidad de Burgos, 491 pp.

Ollé, A., Mosquera, M., Rodríguez, X.P., Lombera-Hermida, A., García-Antón, M.D., García-Medrano, P., Peña, L., Menéndez, L., Navazo, M., Terradillo,s M., Bargalló, A., Márquez, B., Sala, R. and Carbonell, E. 2013. The Early and Middle Pleistocene technological record from Sierra de Atapuerca (Burgos, Spain). Quaternary International, 295, 138-167.

Ortega, A.I. 2009. Evolución geomorfológica del Karst de la Sierra de Atapuerca (Burgos) y su relación con los yacimientos pleistocenos que contiene. Ph.D. Thesis, University of Burgos, 627 pp.

Ortega, A.I., Benito-Calvo, A., Pérez-González, A., Porres, A. and Martín, M.A. 2010. Applying Electrical Resistivity Tomography to the identification of endokarstic geometries in the Pleistocene Sites of the Sierra de Atapuerca (Burgos, Spain). Archeological Prospection, 17, 233-245.

Ortega, A.I., Martín, M.A. and Grupo Espeleológico Edelweiss 2012. Cuevas de Atapuerca: una visión de la mano del Grupo Espeleológico Edelweiss. Diputación Provincial de Burgos, $271 \mathrm{p}$.

Ortega, A.I., Benito-Calvo, A., Pérez-González, A., Martín, M.A., Pérez, R., Parés, J.M., Aranburu, A., Arsuaga, J.L., Bermúdez de Castro, J.M. and Carbonell, E. 2013. Evolution of multilevel caves in the Sierra de Atapuerca (Burgos, Spain) and its relation to human occupation. Geomorphology, 196, 122-137.

Ortega, A.I., Benito-Calvo, A., Pérez-González, A., Carbonell, E., Bermúdez de Castro, J.M. and Arsuaga, J.L. 2014. Atapuerca Karst and its Palaeoanthropological Sites. In F. Gutiérrez, M. Gutiérrez (eds.) Landscapes and Landforms of Spain, World Geomorpholog Landscapes, 101-111.

Palmer, A.N. 2007. Cave Geology. Dayton, Ohio, Cave Books, $454 \mathrm{p}$.

Palombo, M.R. 2013. What about causal mechanisms promoting early hominin dispersal in Eurasia? A research agenda for answering a hotly debated question. Quaternary International, 295, 13-27.

Parés, J.M. and Pérez-González, A. 1995. Paleomagnetic ages for hominids at Atapuerca Archaeological site, Spain. Science, 269, 830-832.

Parés, J.M., Pérez González, A., Rosas, A., Benito, A., Bermúdez de Castro, J. M., Carbonell, E. and Huguet, R. 2006. Matuyama-age lithic tools from the Sima del Elefante site, Atapuerca (northern Spain). Journal of Human Evolution, 50, 163-169.

Parés, J.M., Pérez-González, A., Arsuaga, J.L., Bermúdez de Castro, J.M., Carbonell, E. and Ortega A.I. 2010. Characterizing sedimentary history of cave deposits, using archaeomagnetism and rockmagnetism, Atapuerca (N Spain). Archeometry, 52, 882-898.

Parés, J.M., Arnold, L., Duval, M., Demuro, M., PérezGonzález, A., Bermúdez de Castro, J.M., Carbonell, E. and, Arsuaga, J.L. 2013. Reassessing the age of Atapuerca-TD6 (Spain): new paleomagnetic results. Journal of Archaeological Science, 40, 4586-4595.

Parés, J.M., Ortega, A.I., Benito-Calvo, A., Aranburu, A., Arsuaga, J.L., Bermúdez de Castro, J.M., and Carbonell, E., 2016, Paleomagnetic constraints on the Atapuerca karst development (N Spain), In: Feinberg, J., Gao, Y., and Alexander, E.C., Jr., eds., Caves and Karst Across Time: Geological Society of America Special Paper 516, p. 285-300; doi:10.1130/2016.2516(22).

Pérez-González, A., Parés, J.M., Carbonell, E., Aleixandre,T., Ortega, A.l., Benito, A. and Martín, M.A. 2001. Géologie de la Sierra de Atapuerca et stratigraphie des remplissages karstiques de Galería et Dolina (Burgos, Espagne). L'anthropologie, 105, 27-44.

Pérez, R. 2011. Unificación de la información cartográfica del entorno de los yacimientos de la Sierra de Atapuerca (endokarst y exokarst). Instauración de red geodésica y base para el desarrollo de un sistema de información geográfica. Proyecto Fin de Carrera, Univ. Politécnica, Valencia.

Pineda, A. and Arce, J.M. 1997. Mapa Geológico de España, E 1:50.000, Hoja $n^{\circ} 200$ (Burgos), Serie Magna. IGME, Madrid.

Rodríguez, J., Burjachs, F., Cuenca-Bescós, G., García, N., Made, J.v.d., Rosas, A., Pérez-González, A., Blain, H.A., Expósito, J.M., López-García, J.M., García-Antón, M., Allue, E., Cáceres, I., Huguet, R., Mosquera, M., Ollé, A., Rosell, J., Parés, J.M., Rodríguez, X.P., Díez, C., Rufes, J., Sala, R., Saladié, P., Vallverdú, J., Bennasar, M.L., Blasco, R., Bermúdez de Castro, J,M. and, Carbonell, E. 2011. One million years of cultural evolution in a stable environment at Atapuerca (Burgos, Spain). Quaternary Science Reviews, 30, 1396-1412.

Rodríguez-Gómez, G., Mateos, A., Martín-González, J.A., Blasco, R., Rosell, J. and Rodríguez, J. 2014. Discontinuity of Human Presence at Atapuerca during the Early Middle Pleistocene: A Matter of Ecological Competition? Plos One, 9(7): e101938. doi:10.1371/journal.pone.0101938.

Rosas, A., Huguet, R., Pérez-González, A., Carbonell, E., Bermúdez de Castro, J.M., Vallverdú, J., Made, J.v.d., Allué, E., García, N., Martínez, R., Rodríguez, J., Sala, R., 
Saladie, P., Benito, A., Martínez, C., Bastir, M., Sánchez, A. and Parés, J.M. 2006. The "Sima del Elefante" cave site at Atapuerca (Spain). Estudios Geológicos, 62, 327348.

Vallverdú, J., Courty, M.A., Carbonell, E., Canals, A. and
Burjachs, F. 2001. Les sédiments d'Homo antecessor de Gran Dolina, (Sierra de Atapuerca, Burgos, Espagne). Interprétation micromorphologique des processus de formation et enregistrement paléoenvironnemental des sédiments. L'anthropologie, 105, 45-69.

Recibido: marzo 2015

Revisado: mayo 2015

Aceptado: septiembre 2015

Publicado: marzo 2018 
